\title{
Finite Element Analysis of Three Methods for Microwave Heating of Planetary Surfaces
}

\author{
Edwin C. Ethridge ${ }^{1}$ \\ NASA Marshall Space Flight,Huntsville, AL, 35812 \\ and \\ William Kaukler ${ }^{2}$ \\ University of Alabama Huntsville, Huntsville, AL, 35899
}

\begin{abstract}
In-Situ Resource Utilization will be Ground Breaking technology for sustained exploration of space. Volatiles are present in planetary regolith, but water by far has the most potential for effective utilization. The presence of water at the lunar poles and Mars opens the possibility of using the hydrogen for propellant on missions beyond Earth orbit. Likewise, the oxygen could be used for in-space propulsion for lunar ascent/descent and for space tugs from low lunar orbit to low Earth orbit. Water is also an effective radiation shielding material as well as a valuable expendable (water and oxygen) required for habitation in space. Because of the strong function of water vapor pressure with temperature, heating regolith effectively liberates water vapor by sublimation. Microwave energy will penetrate soil and heat from within, much more efficiently than heating from the surface with radiant heat. This is especially true under vacuum conditions since the heat transfer rate is very low. The depth of microwave penetration is a strong function of the microwave frequency and to a lesser extent on regolith dielectric properties. New methods for delivery of microwaves into lunar and planetary surfaces is being prototyped with laboratory experiments and modeled with COMSOL MultiPhysics. Recent results are discussed.
\end{abstract}

\section{Introduction}

$\mathrm{W}$ ater is one of the most plentiful compounds in the Universe. Our Moon has large quantities of water cryotrapped at the poles. Mars also has vast quantities of water not only at the poles but also at lower latitudes. Significant quantities of water are present on several moons of Jupiter (Europa, Ganymede, and Callisto), Saturn (Enceladus), Neptune (Titon), and possibly on the Martian moon Phobos.

In 1905 a Scientific American article speculated that there was water at the poles of the moon ${ }^{1}$. But, when the Apollo missions returned lunar soil samples, "no water" was present. In 1994, the SDI-NASA Clementine spacecraft mapped the surface of the moon. The microwave radio signals from shadowed craters at the lunar south pole were consistent with the presence of water. In 1998 neutron spectrometer data from Prospector showed high hydrogen concentrations at the poles. On October 9, 2009 the Lunar Crater Observation and Sensing Satellite (LCROSS) crashed into a crater at the south pole. LCROSS observations proved that there are very large quantities of water at the lunar poles.

The presence of water on planetary bodies is of high importance. Water and oxygen are required expendables for extended human exploration. Estimates from architectural studies of a lunar outpost indicated that one ton of water and one ton of oxygen would be needed each year of operation. Use of in-situ water would greatly reduce costs for manned exploration. Water is also recognized for its radiation protection potential. It is only slightly less effective as a radiation shield than polyethylene. The most significant potential application for in-situ water is as a source for $\mathrm{H}$ and $\mathrm{O}$ for use as in-space propellant. Lunar water probably will be the first in-situ resource material that gains significant utilization for exploration activities. The use of ISRU hydrogen for a Mars mission would greatly reduce the required launch mass from Earth. The oxygen would also find use for in-space propellant for lunar ascent/descent as well as for low lunar orbit to low Earth orbit transportation. The dramatic impact by LCROSS on the water laden creater at the lunar south pole proved there is tremendous quantity of water frozen in the polar regolith. In a NASA Science News article of March 18, 2010, Paul Spudis remarked on the LCROSS findings:

"If you converted those craters' water into rocket fuel, you'd have enough fuel to launch the equivalent of one space shuttle per day for more than 2000 years.

\footnotetext{
${ }^{1}$ Materials Scientist, Materials \& Processes Lab., EM41, AIAA Member.

${ }^{2}$ Assistant Research Professor in Chemistry, AIAA non-Member.
} 


\section{Background}

For seven years we have been conducting applied research associated with the use of microwaves for in-space applications. In particular we are developing the use of microwaves for heating/melting regolith and for the extraction of volatiles (especially water) from planetary surfaces. Other volatiles may also be extracted by microwave processes. But, our discussions in the remainder of this paper will concentrate on water extraction since it has the most immediate potential for use.

Microwave processing has several advantages. The thermal conductivity of lunar regolith (in a vacuum or near vacuum) during Apollo shows that regolith is an excellent thermal insulator. If sunlight is directed onto the lunar surface, it would only heat a thin top layer of regolith. And since the heat flow into the regolith is very slow, this would not be an effective method for heating the underlying regolith to temperatures needed for extraction of volatiles.

Microwave energy will penetrate the regolith heating from the inside. The microwave penetration depth is dependent on frequency and on the dielectric properties of the regolith. The absorbed microwave energy heats the regooith which subsequently heats the water ice which sublimes to water vapor and percolates through the regolith to be captured by a cold trap. Since the regolith can be heated in situ, excavation may not be required. The generation of hazardous lunar dust could be minimized. At the cryogenic temperatures of the lunar poles, the frozen permafrost could be as hard as granite making excavation difficult. With microwave water extraction, complex mechanical operations to dig the regolith and processing chambers proposed for other extraction processes might not be required.

\section{A. Proof of Principle}

In prior work, we demonstrated that microwave energy will couple to cryogenically cooled lunar soil simulant (JSC-1A) containing 1\% water-ice. ${ }^{2}$ The permafrost simulant was contained in a fused silica vessel, our "moon in a bottle", for this proof of principle experiment (Figure 1). As the cold permafrost simulant was heated from cryogenic (LN2) temperatures with $2.45 \mathrm{GHz}$ microwaves, the water ice sublimed (less than 2 minutes) at temperatures well below $0^{\circ} \mathrm{C}$. This water vapor escaped the simulant and traveled along the line connected to a vacuum pumping station. An inline cold trap between the two captured virtually all of the liberated water. This proof of principle experiment validated the concept of microwave heating of lunar regolith simulant to extract water. It was demonstrated that microwaves $(2.45 \mathrm{GHz})$ will couple with lunar soil simulant (that does not contain nanophase iron) at cryogenic temperatures. The resulting heating of the simulant caused the water ice to rapidly sublime and was collected in a cold trap with high efficiency.

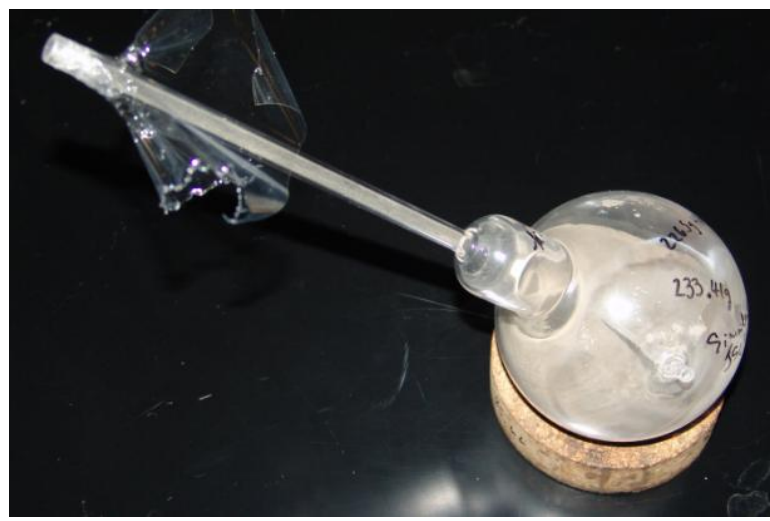

Figure 1. Our "Moon in a Bottle" fused silica (microwave transparent) containment vessel used to hold the lunar regolith permafrost simulant while a vacuum is pulled on the glass tube. The regolith permafrost simulant was cooled with liquid nitrogen. The sample was placed in a domestic microwave oven to heat the simulant. 
Heating of the regolith depends on absorption of the microwave energy. The extent of microwave absorption is dependent on several factors. The penetration depth is greatly affected by the microwave frequency. Long wavelengths (lower frequencies) penetrate deeper than short wavelength (higher frequencies). Knowledge of the dielectric properties is required to calculate the penetration depth and to model soil heating and water extraction.

\section{B. Laboratory Experiments}

We have been developing water extraction methods both by experimentation and with numerical modeling. The first system was prototyped to get an understanding of how a microwave system might be constructed and to test system components, see Figure 2a. It directs microwaves $(2.45 \mathrm{GHz})$ down directly into the lunar regolith simulant. A magnetron generates the microwaves that pass though a waveguide to a high gain horn antenna with good forward gain. A three stub tuner was used for impedance matching when necessary. Heating within the simulant bed was measured with Luxtron fluoro-optic thermometry with 4 fiberoptic sensors. The temperature increase is consistent with calculations of attenuation.
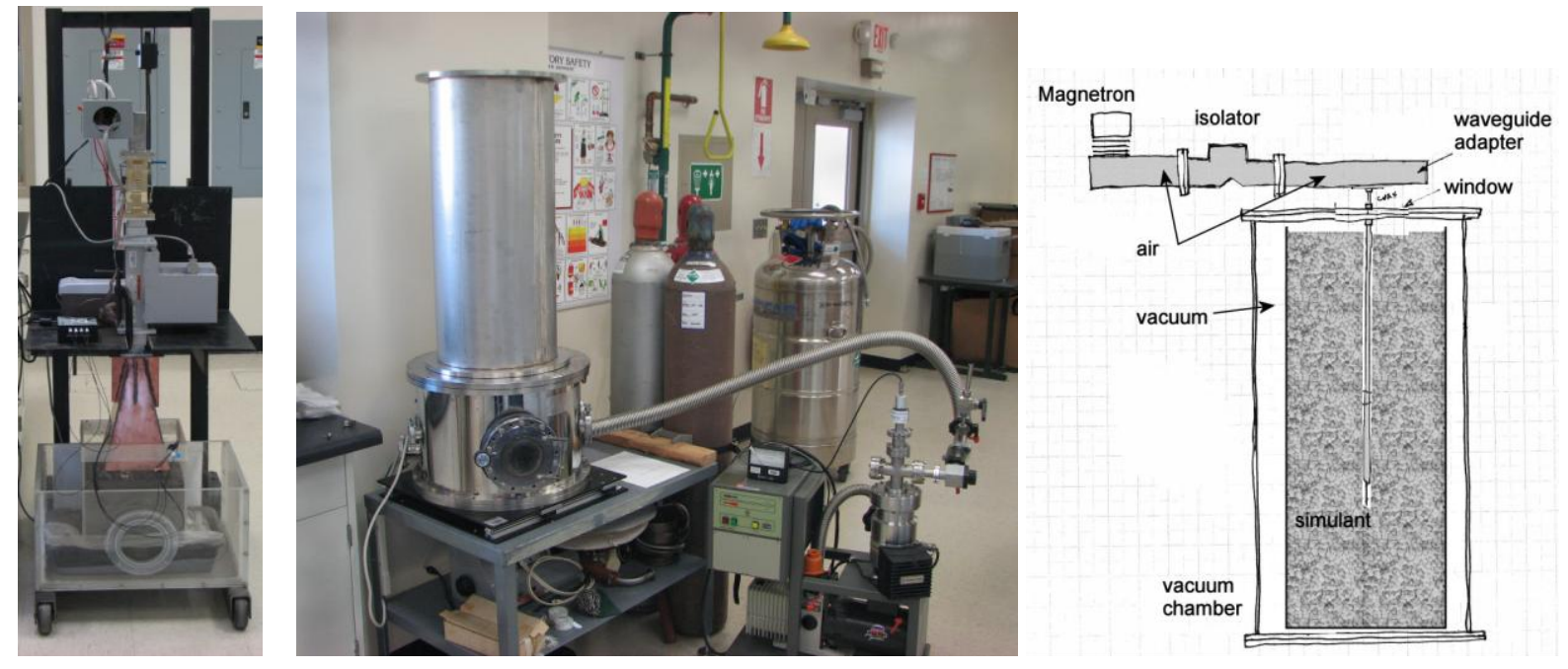

Figure 2. a) On the left is our laboratory setup for directing microwave energy into a planetary surface analogue with a $2.45 \mathrm{GHz}$ microwave horn. b) In the middle, is a laboratory setup of a vacuum system for testing of the extraction of water through a borehole from 1 meter below the top surface of a bed of regolith simulant permafrost. c) On the right is a schematic drawing of the experimental setup in $b$.

In another laboratory experiment, we are evaluating new methods for getting microwaves deep below the surface. A vacuum system is connected to a vacuum ring attached to a 1 meter chamber (Figure $2 \mathrm{~b}$ ). Microwave energy is delivered down 1 meter thorough the regolith where the microwave energy heats the surrounding regolith simulant containing ice and other volatiles. The water vapor and other volatile species that are liberated will be measured with a mass spectrometer. A schematic drawing in Figure $2 \mathrm{C}$ shows the inside of the vacuum system. This vacuum system has been installed in a low temperature $\left(<-77^{\circ} \mathrm{C}\right)$ laboratory freezer. These experiments are in progress.

Temperatures measured at different depths in the simulant confirm that the microwave energy does, in general, attenuate according to Beer's Law as it penetrates the simulant. Experiments at low temperatures in a vacuum are difficult and measurements are limited to a few (4) locations since fiber optic measurements are required. Interpretation of such limited numbers or measurements is also difficult. Likewise, experimental setups for experiments at different microwave frequencies will be both time consuming and expensive. For this reason modeling software is being used to perform parametric numerical calculations of hypothetical experiments. 


\section{Permittivity and Permeability Property Measurements}

In order to perform numerical analysis and regolith heating calculations, the dielectric properties are needed. Our first measurements utilized the transmission line filled waveguide method. Microwave waveguide fixtures had to be designed and fabricated, see Figure 3a. The waveguides were connected to coaxial instrumentation signal cables to the network analyzer. An Agilent programmable network analyzer (PNA, model number E8361A with A.06.04.30 firmware) from the Microwave-millimeter Instrument Characterization Laboratory at the MSFC Materials \& Processes Laboratory was utilized for the measurement of complex electric permittivity and magnetic permeability. A dedicated software package (Agilent 85071 version E2.24.02 Materials Measurement Software from Innovative Solutions Inc.) takes the data from the analyzer and fits it to appropriate models to determine the desired properties.

The second measurement method (Figure $3 b$ ), the resonant cavity perturbation method ${ }^{3,4}$, has been under development by Agilent and we worked with Agilent for its implementation in our laboratory. This resonant method is appropriate for measuring small samples. It is especially suitable for measurements of Apollo lunar soil samples, which we hope to have the opportunity to measure. Another advantage is the sensitivity of the method to the complex dielectric constant. This method is claimed to be more accurate than the transmission line technique for permittivity of low-loss materials such as lunar regolith simulant.
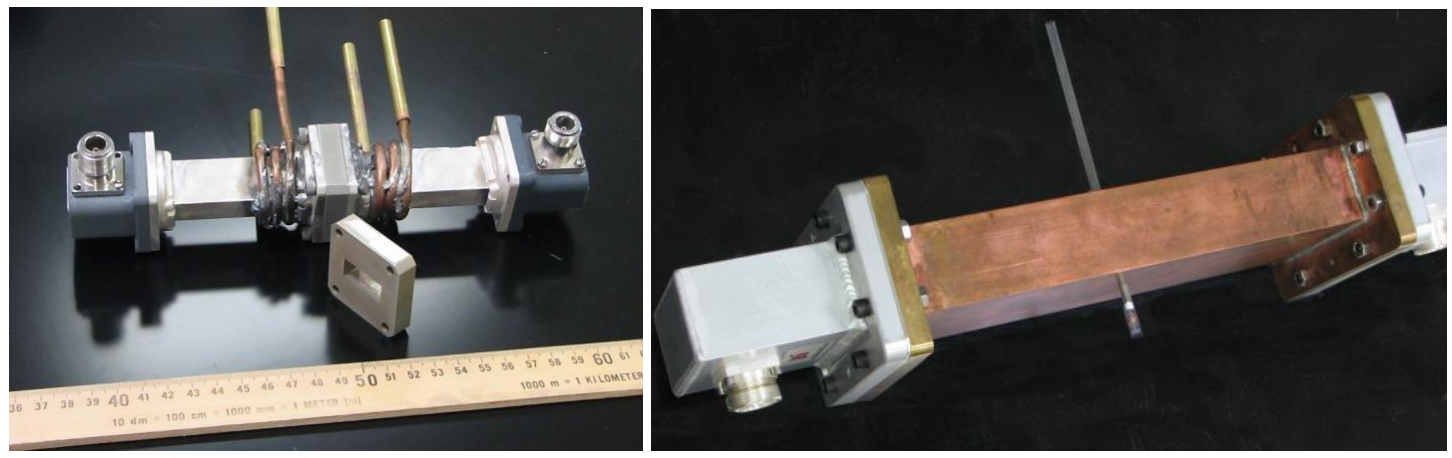

Figure 3. a) On the left, an X-band $(10 \mathrm{GHz})$ test fixture for dielectric measurements using the transmission/reflection filled waveguide method. The sample is placed in a sample holder and mounted in the center of the fixture. Mica sheets are used to contain the simulant power. b) On the right, is an S-band (2.45 $\mathrm{GHz}$ ) resonant cavity fitted with the coax to waveguide adapters at each end. Through the midpoint of the waveguide, a sample holding tube passes through the waveguide. Waveguide coupling apertures not shown, are sized empirically for these measurements. The copper waveguide is about one foot long.

It has been reported, that Apollo lunar regolith couples very well with microwave energy ${ }^{5,6}$. Lunar regolith contains very small nano-phase metallic iron (n-Fe) spheres in the surface layer of regolith particles. It is said that $\mathrm{n}$-Fe causes significant coupling with microwaves which aids the heating of lunar soil. For this reason the Lunar Simulant Workshop ${ }^{7}$ identified the importance of replicating the microwave dielectric properties of lunar soil as with lunar simulants. The workshop concluded that a daughter simulant derived from JSC-1a should be developed to simulate the effect of nano-phase iron. Other investigations (Orbitec, Inc, Plasma Processes, etc.) have produced daughter simulants containing agglutinates and some n-Fe.

We have measured the complex electric permittivity and magnetic permeability of several lunar soil simulants including those that contain Fe. The baseline standards are the lunar mare simulants JSC-1 and JSC-1A. A daughter simulant, JSC-1A AGGL, was produced by Orbitec, Inc. which contains agglutinates with nano-phase elemental iron in the thin surface layer. Transmission microscopy of the samples show spherical nano-phases that look very much like those in lunar soil. The USGS NU-LHT lunar highlands and Orbitec's JSC-Mars-1A simulant were also measured. Also, to evaluate the possible effects of elemental iron on the permittivity and permeability, we made samples of JSC-1A containing 1 weight $\%$ iron power in two different forms. Our first is 
JSC-1A simulant with 200 micrometer sized metallic iron powder mechanically mixed with JSC-1A. Our second daughter simulant is JSC-1A mixed with Carbonyl Iron Powder (CIP) with 3 to 5 micrometer size particles. Since the iron in these mixtures is not contained within the surface layers of the regolith particles, we recognize that these daughter simulants are different from the lunar regolith containing nano-phase iron.

Last year we reported our dielectric property data for these lunar regolith simulants. ${ }^{8}$ For our measurements at room temperature and $2.45 \mathrm{GHz}$, we got a range of values for the dielectric constant (e') of JSC-1A from 3.9 to 4.2. All of our JSC-1A derived simulants including those containing iron particles were basically in the same range (3.8 to 4.2) being essentially no different from the JSC-1A. Our measurements for NU-LHT and JSC-Mars1A did have significantly lower values for e', 3.3 to 3.4. The imaginary part of permittivity (e") was more difficult to measure and ranged from 0.6 to 0.9 for all JSC-1A simulants. All of our permeability measurements at room temperature, were very similar (u' 1.00 to 1.01 and u" from 0.001 to 0.003 ) for all of the simulants.

A few measurements were performed for $10 \mathrm{GHz}$ at room temperature. For the JSC-1A simulants, e' ranged from 3.2 to 4.1. Compared to the $2.45 \mathrm{GHz}$ measurements there appears to be a more significant difference between the e" measurements for simulants without iron additions compared with those with iron additions. For JSC-1 A the value of e" is 0.08 . While for JSC-1A with iron particles, e" values of 0.2 and 0.5 were measured. The values for permeability were $\mathrm{u}^{\prime}=1$, and $\mathrm{u}^{\prime \prime}=0.001$ for all simulants at $10 \mathrm{GHz}$.

Our measurements at lower temperatures $(\sim-77 \mathrm{C})$ indicated that the value for e' is a little lower than room temperature values, while e", $u^{\prime}$, and u" are not measurably different from room temperature. Our general conclusion is that the permittivity and permeability values for regolith simulants are within $5 \%$ of the values for JSC-1 A at room temperature to temperatures down to $200 \mathrm{~K}$. The values are somewhat lower at sub ambient temperatures and increase for temperatures above ambient. This agrees with some unpublished data that has come to the attention of the author. For the subsequent COMSOL calculations in this paper, the permittivity and permeability will be considered constant. It is generally accepted that the dielectric constant will increase significantly at higher temperatures. This would greatly increase the microwave heating effect at higher temperatures.

\section{COMSOL FEM Modeling}

COMSOL Multiphysics FEM analysis has been used to calculate the microwave heating of regolith from cryogenic temperatures. COMSOL solves the differential equations for the physics in the problem using sophisticated built in solvers that are optimized for each particular physics. Our numerical modeling of regolith heating with COMSOL utilizes two physics software modules. First, the RF Module is used to calculate the penetration of microwaves into a volume of regolith. Depending on the model geometry, either a 3-Dimensional or 2-D axi-symmetric geometry is used for these calculations. The microwave frequency is fixed for each calculation. Nominal dielectric properties measured for JSC-1A lunar soil simulant and other materials properties (Tables I and II) were used for the calculations. The model design was tested and tweaked with corrections to the model geometry, modifications to the mesh, and numerous trial calculations until calculations converged. The next step in the model development is the addition of the heat flow physics. The calculated microwave energy absorbed by the regolith simulant in the RF module is used by the Heat Transfer module to calculate regolith heating and temperatures.

Certain assumptions were made regarding the temperature of sublimation of water from the regolith. McCoustra and Williams ${ }^{9}$ studied the adsorptionldesorption of water on dust grains modeling the interaction in interstellar molecular clouds with an ultra high vacuum system (1.5E-10 torr). Rapid desorption (sublimation) of water was observed to begin at $130 \mathrm{~K}$. This corresponds precisely to the phase boundary for water sublimation at that temperature and pressure. ${ }^{10}$ Also, according to the equation for the phase boundary for sublimation of water ice, at $200 \mathrm{~K}$ water sublimes at a pressure of 1 millitorr. Throughout this paper we will assume that once regolith reaches $200 \mathrm{~K}$, water will rapidly sublime. This assumes that the ambient pressure is less than 1 millitorr. If the pressure is lower, then water will rapidly sublime at a lower temperature. Three different microwave heating methods will be described in the remainder of the paper. 


\section{Microwave Oven Heating of Simulated Lunar Regolith}

The first COMSOL Multiphysics model to be discussed in this paper is for microwave heating of JSC-1A regolith simulant with a microwave oven at $2.45 \mathrm{GHz}$ in air starting at room temperature. An example from the COMSOL Model Library was adapted for this model. The sample being heated was changed to a cylinder of material with properties representative of JSC-1A at room temperature, See Table I.

Table I. Materials Values for "Room Temperature" Calculations

$\begin{array}{lccc}\text { Thermal Conductivity } & \mathrm{k} & \text { (dry sand) } & 0.35 \mathrm{~W} / \mathrm{m} * \mathrm{~K} \\ \text { Specific Heat } & \mathrm{Cp} & 0.85 \mathrm{KJ} / \mathrm{Kg}^{*} \mathrm{~K} \\ \text { Density } & \rho & \text { measured } & 1.66 \mathrm{~g} / \mathrm{cm}^{3} \\ \text { Electrical conductivity } & \sigma & 0 \\ \text { Permittivity } & \mathrm{e},-\mathrm{e} * \mathrm{j} & 4-0.1 \mathrm{j} \\ \text { Permeability } & \mathrm{u},-\mathrm{u} " * \mathrm{j} & 1-0.01 \mathrm{j}\end{array}$

In the absence of permittivity and permeability values at temperatures above room temperature, the nominal room temperature values in Table I were used. The electrical conductivity is effectively zero. The thermal conductivity and specific heat of dry sand and our measurement of the density of JSC-1A were used for the thermal calculations. The initial condition for temperature (To) for this first example is $300 \mathrm{~K}$. Experiments have shown that our $1 \mathrm{KW}$ (electrical input) laboratory oven will produce $500 \mathrm{~W}$ of thermal heating (50\% efficient) determined from calorimetric measurements, so $500 \mathrm{~W}$ was used for the power in the calculations. Figure 4 has a sequence of three sets of isotherms through the cylindrical sample $(6 \mathrm{~cm}$ diameter, $15 \mathrm{~cm}$ tall $)$ for three heating times. The heating is very non-uniform with large thermal gradients in the sample. After 1 hour, portions of the sample heat to over 700K. Not knowing the high temperature dielectric properties of JSC-1A, the calculations cannot be precise, but, these results are consistent with our experience. We have heated JSC-1 A until portions of JSC-1A samples enter thermal runaway and melt the simulant.
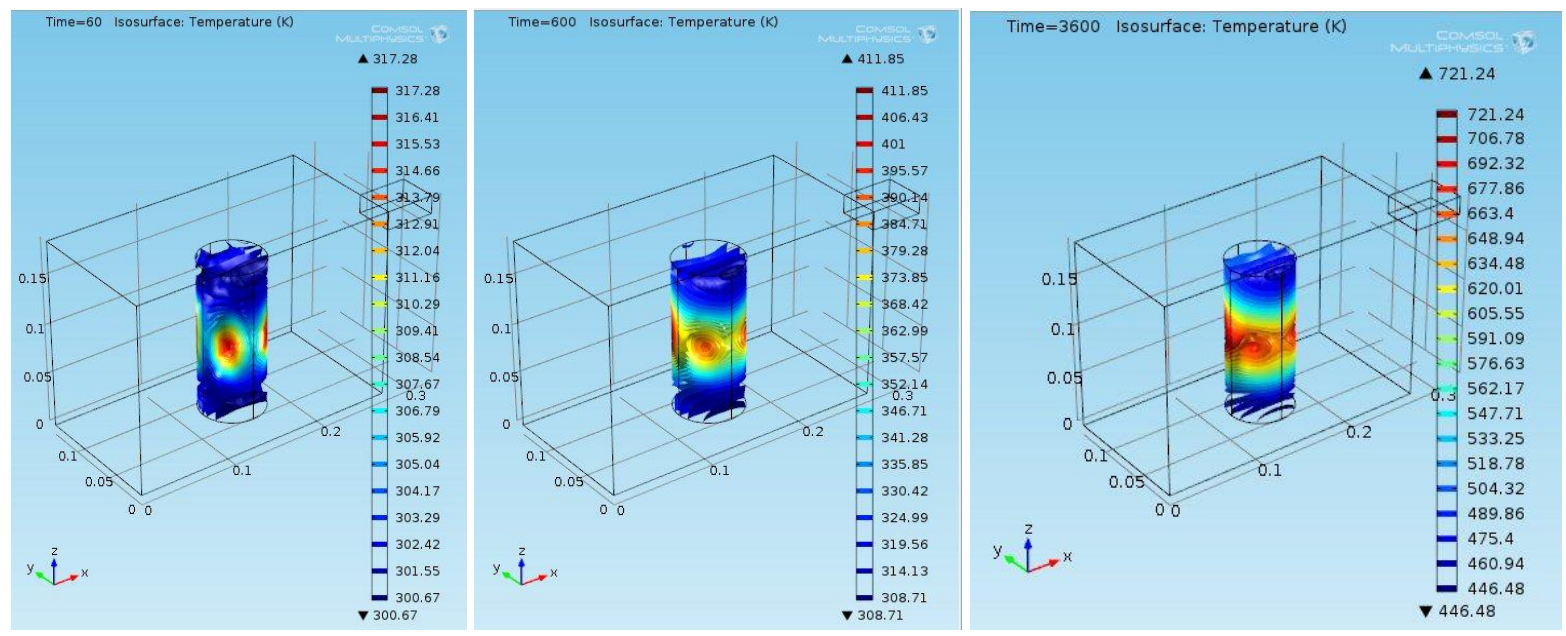

Figure 4. COMSOL Multiphysics FEM analysis of microwave oven heating of a cylinder of lunar regolith simulant, JSC-1A from room temperature (300K). The three heating times from left to right are, 1 minute, 10 minutes, and 1 hour.

The uneven heating is caused by an extremely non-uniform electric field intensity within the oven, three slices through the oven are shown in Figure 4. This is why food is rotated within a microwave oven. The low thermal conductivity of the regolith in a vacuum would also further contribute to difficulties with uniform heating. The efficient use of microwave ovens for heating of regolith would require maximizing the design of the oven and perhaps rotation of the sample for more uniform heating. 


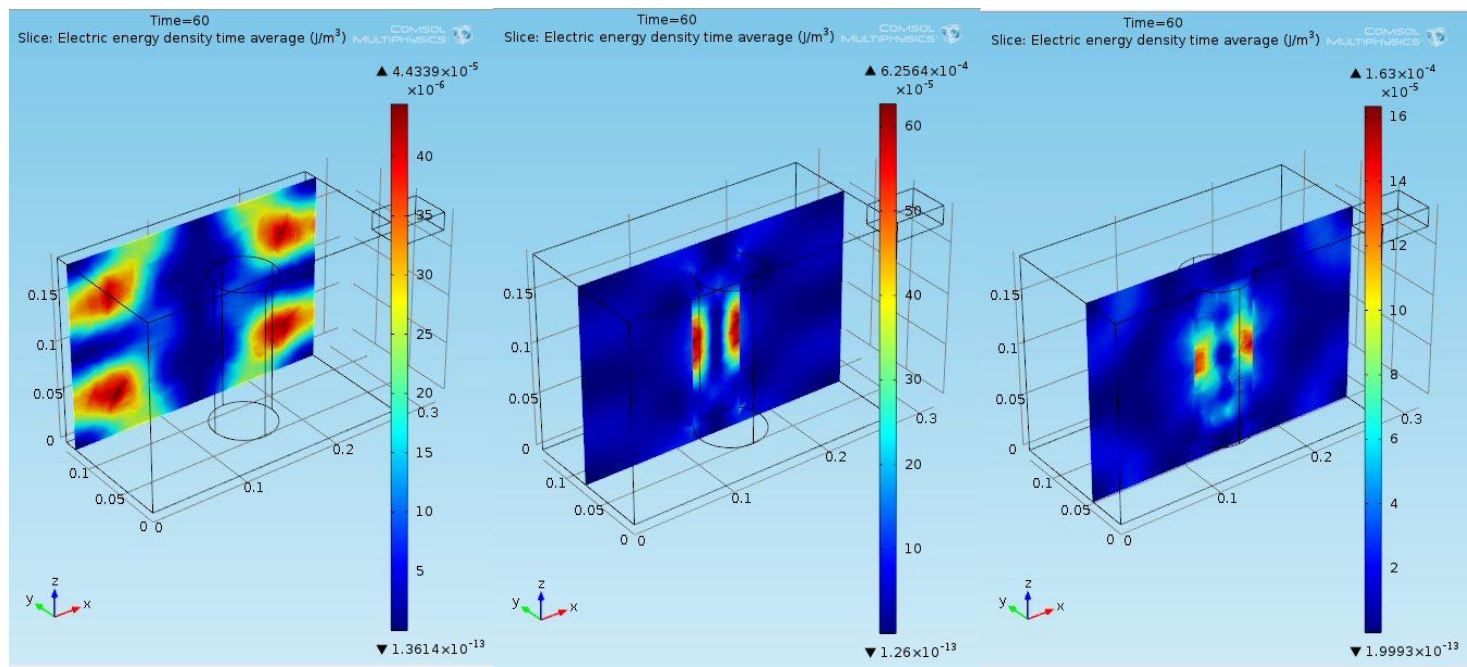

Figure 5. Planar slices through the microwave oven example showing the electric energy density time average $\left(1 \mathrm{E}-13\right.$ to $1 \mathrm{E}-12 \mathrm{~J} / \mathrm{m}^{\wedge} 3$ ) through the oven and within the cylindrical sample.

The next example uses this same microwave oven geometry to illustrate a regolith heating experiment from a planetary surface. A lower power $(50 \mathrm{~W})$ was used for this calculation, since power will be a limited resource in space experiments. The sample size was reduced to a cylinder with a radius and height of $1 \mathrm{~cm}$. The initial temperature was assumed to be $100 \mathrm{~K}$. For this and the remainder of the calculations in this paper, conditions similar to that on planetary surfaces are to be modeled. The Lunar Sourcebook ${ }^{11}$ provides data for the thermal conductivity measured by Apollo with values ranging from 1.5E-4 near the surface to $2.95 \mathrm{E}-4$ (W/cmK) over $2 \mathrm{~m}$ deep. This was correlated to the regolith density. For our calculations, the thermal conductivity is assumed to be $2 \mathrm{E}-4(\mathrm{~W} / \mathrm{cmK})$ since this corresponds to the lunar regolith with a density of $1.66 \mathrm{~g} / \mathrm{cm}^{3}$ which is the density we have measured for JSC-1A. A simple linear relationship fit through the lunar regolith specific heat data of Crews $^{12}$ (see Table II) was used to estimate the temperature dependence (100 to $400 \mathrm{~K}$ ) of the specific heat at the low temperatures. The other regolith properties used for these calculations are also listed in Table II.

Table II. Materials Properties for Calculations starting at 100K.

\begin{tabular}{|c|c|c|c|c|}
\hline Thermal Conductivity & $\mathrm{k}$ & $2 \mathrm{E}-4$ & $\mathrm{~W} / \mathrm{cm} * \mathrm{~K}$ & lunar regolith \\
\hline Specific Heat & $\mathrm{Cp}$ & $2.67 \mathrm{~T}$ & $\mathrm{~J} / \mathrm{Kg} * \mathrm{~K}$ & lunar regolith \\
\hline ensity & $\mathrm{f}$ & 1.66 & $\mathrm{~g} / \mathrm{cm}^{3}$ & densified JSC-1A, measured \\
\hline Electrical conductivit & $y$ & 0 & & ro conductivity \\
\hline ermittivity* & $e^{\prime}-e^{\prime \prime} * j$ & $4-0.1$ & & change JSC- $1 \mathrm{~A}, \mathrm{n}$ \\
\hline ermeability* & $u^{\prime}-u^{\prime \prime} * j$ & $1-0.0$ & & significant change JSC-1A, mea \\
\hline
\end{tabular}

Figure 6 shows 3-D isotherm plots of heating of the small cylindrical sample placed into the same microwave oven model. Again there is localized heating in the sample. Figure 6a shows the sample within the oven. It is quite small compared to the oven, but, our intent was not to redesign the oven. The second view (Figure 6b) shows an enlarged image of the sample heated for 1 minute. The maximum temperature in the cylinder is only $104 \mathrm{~K}$, very little heating from the original 100K. Slices through the oven (Figure 6c) show the localized regions of high electrical energy density. 


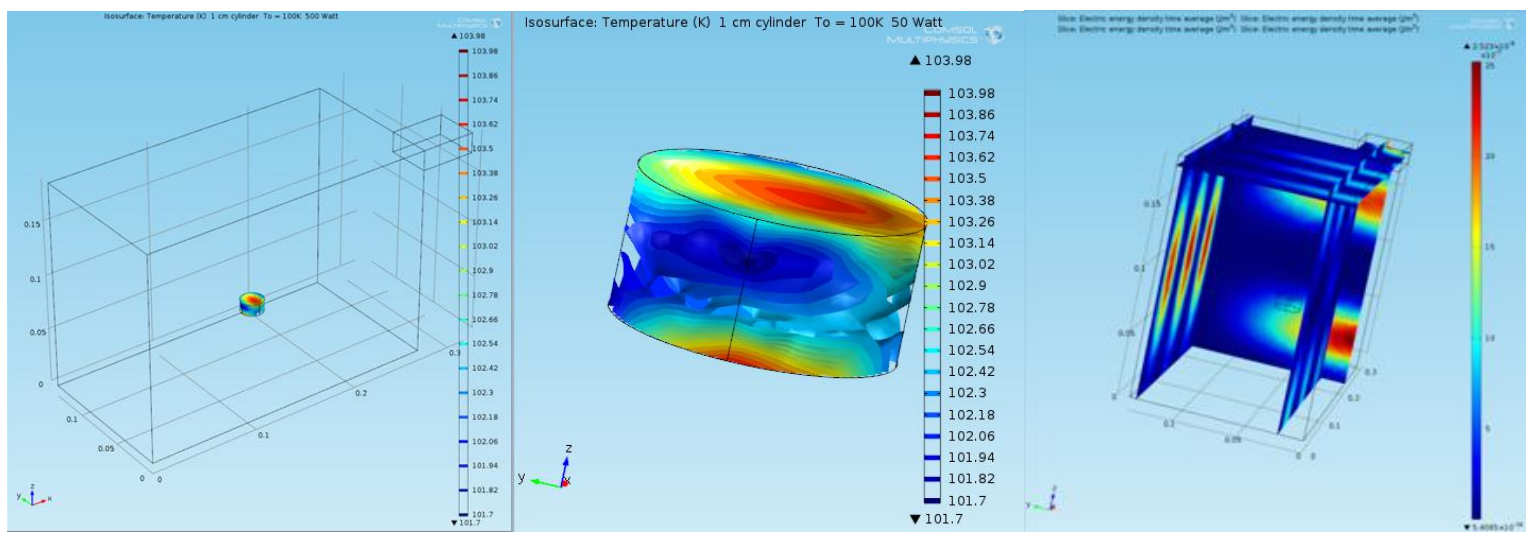

Figure 6. a) The small cylindrical sample within the microwave oven model geometry. b) Magnified view of the sample showing temperature isotherms in the sample and c) Plane slices showing the energy density in the oven.

A series of trial and error adjustments of the sample position and recalculations were performed to reposition the cylinder in a higher energy density region. Each time the sample was moved, the high energy nodes would change. After a few hours of trial and error testing, the cylinder was located at a hot spot shown in Figure 7. The node is close to the magnetron and the energy coupling into the sample was much greater. The energy absorbed and the temperature within the cylinder is much higher ranging from $390 \mathrm{~K}$ to $482 \mathrm{~K}$. The planar slices through the oven illustrate the change in electric energy density distribution.

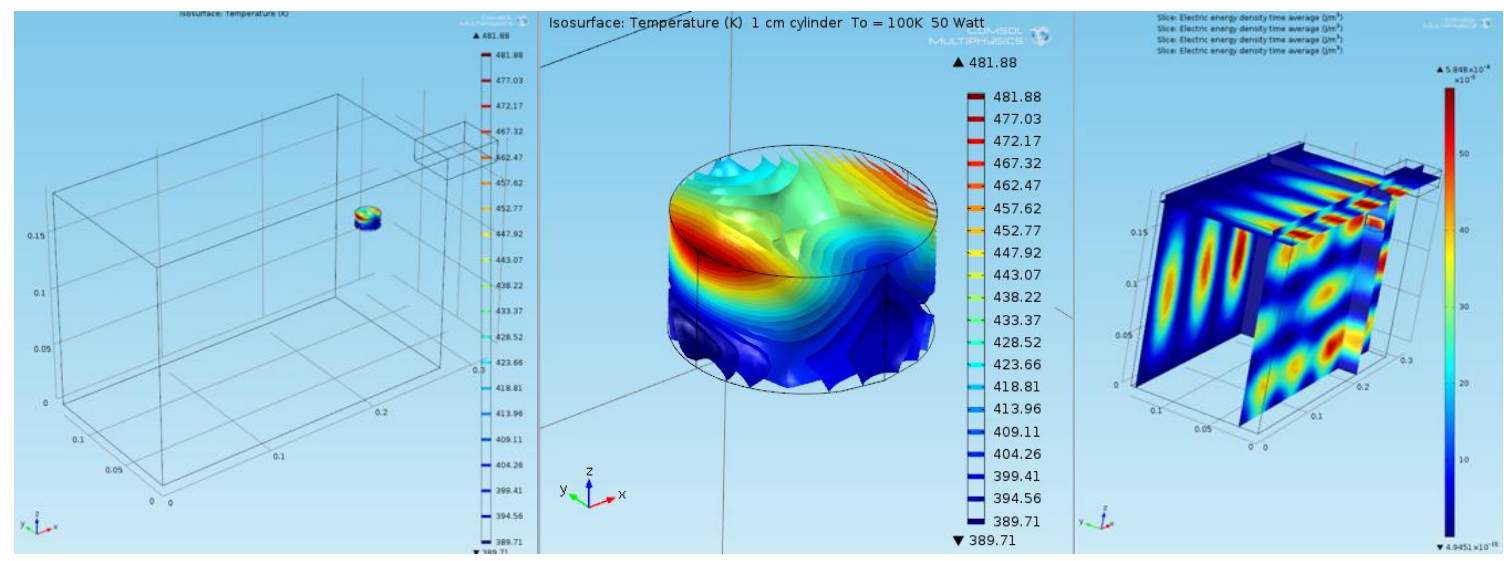

Figure 7. Microwave heating of the cylinder sample after locating within a high energy density region with temperatures in the sample cylinder from 390 to $482 \mathrm{~K}$ after 1 minute of heating.

\section{Beaming Microwaves at Planetary Surfaces with a High Gain Horn}

The next microwave heating example uses a $2.45 \mathrm{GHz}$ high gain microwave horn to deliver microwave energy into a planetary surface such as Mars where water would be close to the surface. The power used for this example is 500 watts and the heating time is 10 minutes. The first image in Figure 8 shows temperature slices with isotherms within the 3-D regolith box (with $25 \mathrm{~cm}$ sides) below the microwave source. Calculated temperatures range from the initial temperature of $100 \mathrm{~K}$ (representative of a lunar polar crater) to over $400 \mathrm{~K}$. The second image shows the $200 \mathrm{~K}$ isotherm. According to our earlier assumption, it is expected that all of the water within the $200 \mathrm{~K}$ isotherm would sublime. 


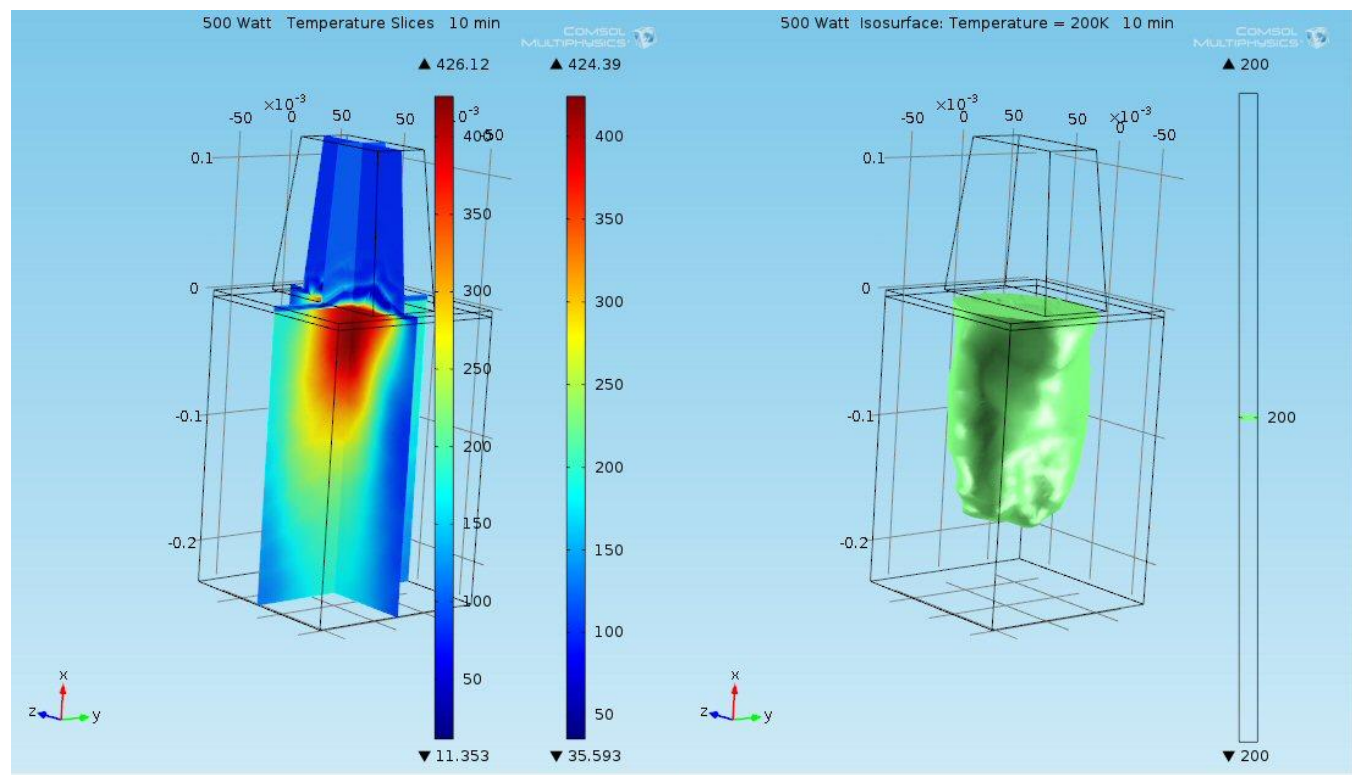

Figure 8. Power of 500 watt, 10 minutes of heating. Plane slices showing the depth of penetration and heating into the surface (on the left). Isotherm at $200 \mathrm{~K}$ illustrating the volume that would be heated sufficiently to completely volatilize the water present in the regolith.

Another example of directing microwave energy into a planetary surface is shown in Figure 9. This could be used for volatiles science of an object such as an asteroid or comet. In this case, a much higher frequency of 24.5 $\mathrm{GHz}$ from a microwave horn is beamed from half a $\mathrm{cm}$ above the surface. For this example the initial temperature is $100 \mathrm{~K}$ and 100 watts of power for 60 minutes heats a $1 \mathrm{~cm}$ volume of regolith. A large portion of the volume is heated to more than $200 \mathrm{~K}$ and the red regions heat to nearly $1500 \mathrm{~K}$. These temperatures would be sufficient to desorb and sublime all volatiles as well as to decompose minerals containing volatile constituents.

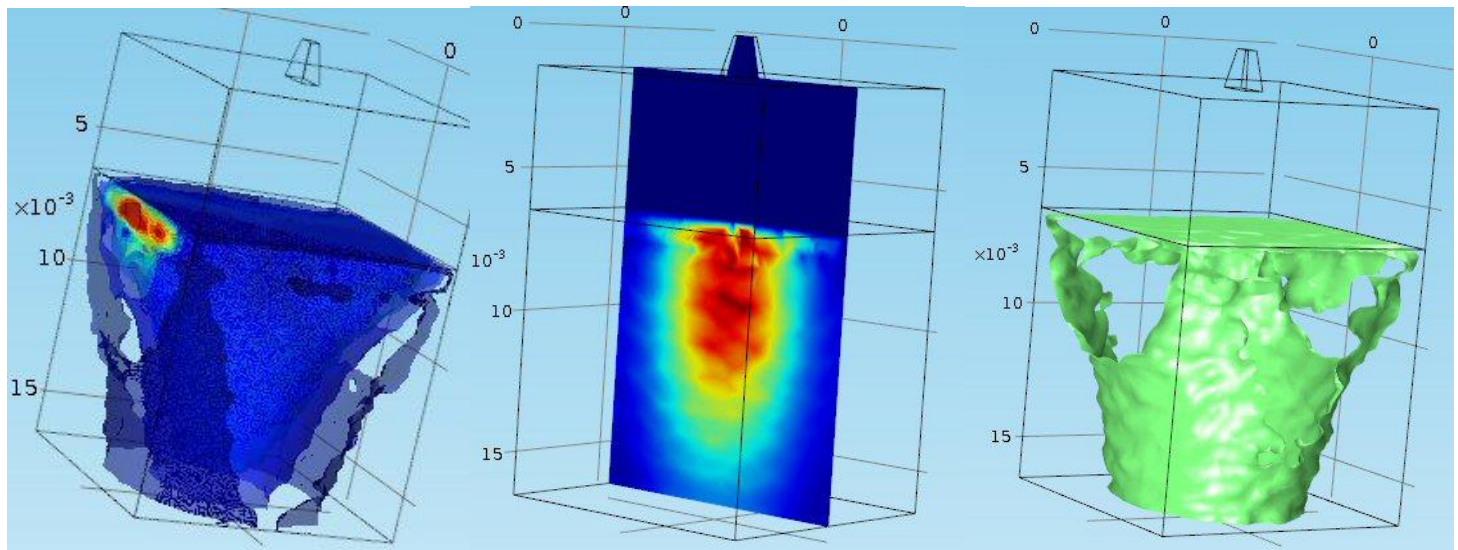

Figure 9. $24.5 \mathrm{GHz}$ microwaves beamed at a planetary surface with a microwave horn from $0.5 \mathrm{~cm}$ away from the surface. Heating within a $1 \mathrm{~cm}$ cube is illustrated. From left to right, a) Isothermal bands of heating within the $\mathrm{cm}$ cube. b) plane slice through the box showing heating from $100 \mathrm{~K}$ to nearly $1500 \mathrm{~K}$, and c) Isotherm at $200 \mathrm{~K}$, volume within the isothermal boundary is hotter than $200 \mathrm{~K}$ and in the vacuum of space and according to the water sublimation phase boundary, all the water should sublime.

\section{Heating in a Borehole at the End of a Probe}

The last example is a simple quarter wavelength monopole antenna at the end of a coax feed line that is used to heat regolith down a borehole. This is the launcher design that is being used to test our laboratory hardware and to test the extraction of water from the bottom of a borehole in regolith simulant. This example also illustrates the need 
to properly design the microwave launchers. We were getting the vacuum system ready to be "safety tested" to verify that we had no leaking of microwave energy from the vacuum chamber into the room. Our initial probe design was connected through an RF bulkhead connector and powered up. In a short time the probe burnt at the connector within the chamber. Our microwave engineer pointed out an error with the launcher design which caused lobbing of the power along the probe. This was verified with COMSOL calculations of the electrical power density along the probe, see Figure 10. After redesigning the launcher, it performs correctly and according to the analysis, the power is delivered into the simulated regolith heating a roughly spherical volume at the end of the launcher. We are also measuring and calculating S-parameters for our microwave launchers. This illustrates how we are also using the COMSOL to test the design of our microwave launchers.

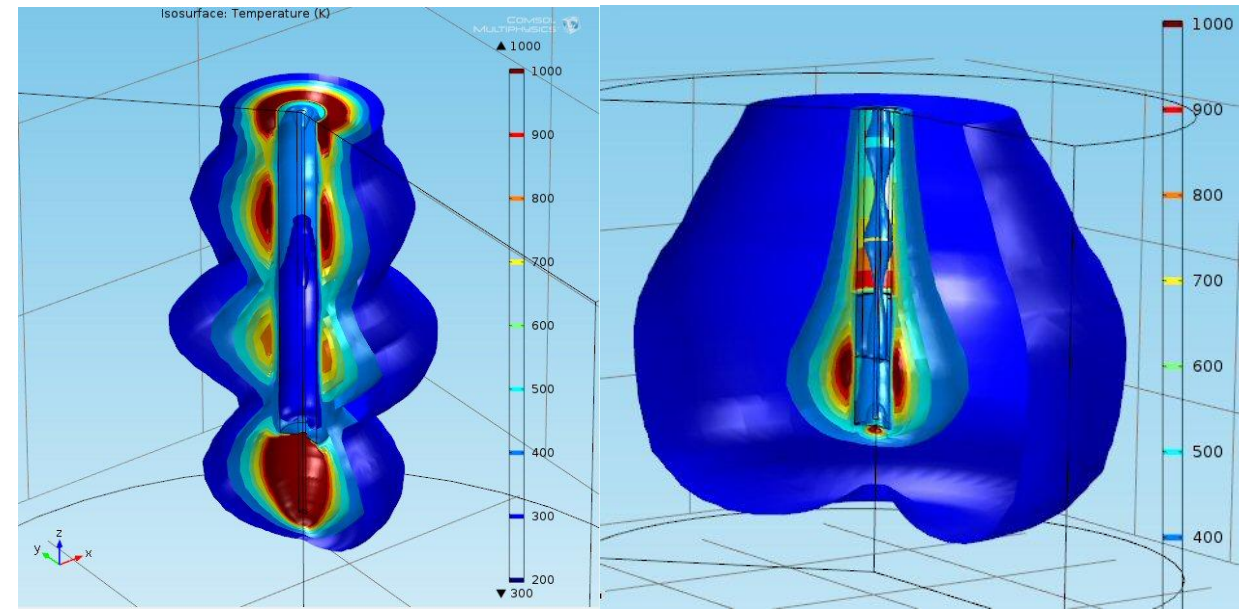

Figure 10. Heating of a monopole launcher with an improperly designed ground plane (on the left) showing lobes along the coax feed line. After correcting the geometry with a correct launcher design, the power effectively radiates into and heats the regolith (on the right).

Calculations of the heating at the tip of the monopole launcher with 100 watts for 1 minute are shown in Figure 11. The Isotherms in the figure range from the starting temperature of $100 \mathrm{~K}$ to the sublimation temperature of water at $200 \mathrm{~K}$. The brown region highlights the volume at temperatures greater than $200 \mathrm{~K}$. After 10 minutes the region heated above $200 \mathrm{~K}$ is more than $10 \mathrm{~cm}$ across.

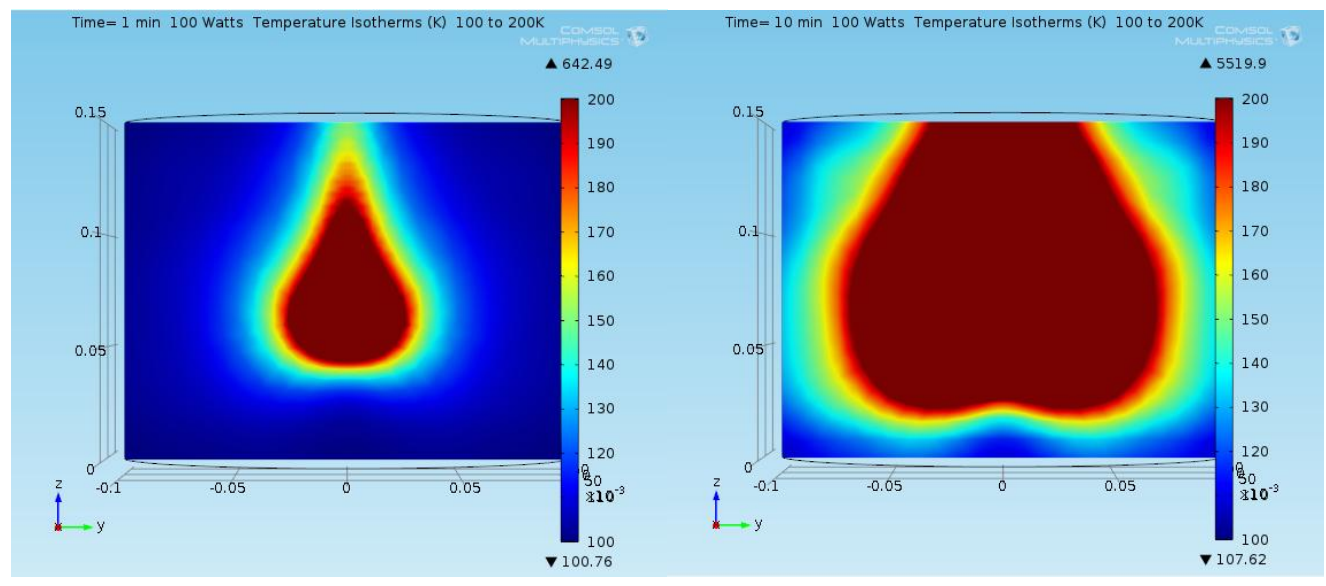

Figure 1. Isothermal plots (from 100 to $200 \mathrm{~K}$ ) at the tip of the monopole launcher after 1 minute (on the left) and 10 minutes (on the right). The brown region is $>200 \mathrm{~K}$, and should be completely devoid of water.

The most important thing learned from the numerical modeling is that one can calculate the heating processes in the soil for different experimental and processing conditions. Multiphysics FEA modeling permits 
the calculation of heating effects depending on specific soil dielectric properties, thermal properties, and the temperature dependence of those properties. The modeling also permits one to calculate the heating effects based on the microwave frequencies, power level, and length of time of heating. It will be much less expensive to perform parametric calculations for preliminary hardware designs of potential space experiments, as well as to perform calculations of potential hypothetical experiments. Once general optimal experimental parameters are known, then more definitive experiments can be performed in our planetary testbed facilities. ${ }^{8}$ This kind of understanding of the physics underlying the microwave heating of regolith to extract volatiles will be extremely important for experiment design and hardware requirements needed to design experiments both in the lab and on planetary surfaces.

\section{Conclusion}

1. COMSOL FEM Multiphysics models have been developed and calculations performed to simulate laboratory experiments. The results are consistent with the observations from the experiments and the microwave energy penetration, absorption, and heating correlate with calculations.

2. Calculations are consistent with experimental observations, that microwaves $(2.45 \mathrm{GHz})$ will couple with lunar regolith simulants at cryogenic temperatures and heat to temperatures where water ice will sublime at lunar and Martian conditions. This occurs even without nano-phase metallic iron particles present in lunar regolith.

3. The range of the dielectric properties of lunar regolith simulants that have been measured have a minor effect on the heating of regolith simulant from cryogenic temperatures to room temperature (300K).

4. COMSOL multiphysics is useful for modeling microwave penetration at different microwave frequencies and microwave heating with processing time permitting the determination of the volume of regolith that is heated sufficiently to completely volatilize the water and other volatiles present in the regolith.

5. COMSOL will be useful for parametric studies of different scenarios for microwave heating of different planetary surfaces for the design of scientific experiments. It will also permit the design of microwave components as well as the determination of hardware requirements for the efficient extraction of water and other volatiles.

\section{Acknowledgments}

We wish to thank NASA Headquarters for the financial support of this Lunar Advanced Science and Exploration Research (LASER) research project. Special thanks to MSFC Management for prior seed funding that permitted us to develop the initial concepts. We also acknowledge Walter Frei at COMSOL for his technical assistance with RF microwave heating and COMSOL models.

\section{References}

${ }^{1}$ Anomous, "Is there life on the moon", Scientific American Volume XCII, No 26, p 511, December 23, 1905.

${ }^{2}$ Ethridge, E. and W. Kaukler, "Extraction of Water from Extraterrestrial Bodies - Dielectric Property Measurements", AIAA 47th Aerospace Sciences Meeting, Orlando, FL, January 5 to 82009.

${ }^{3}$ Kumar, A., S. Sharma, and G. Singh, "Measurement of Dielectric Constant and Loss Factor of the Dielectric Materials at Microwave Frequencies,” Prog. Electromagnetics Res., PIER 69, 47-54, 2007

${ }^{4}$ American Standards for Testing Materials, ASTM A893/A893M-03 "Standard Test Method for Complex Dielectric Constant of Nometallic Magnetic Materials at Microwave Frequencies"

${ }^{5}$ Taylor, L.A. and Meek, T.T., "Microwave sintering of lunar soil: Properties, theory, and practice", J. Aerospace Engr., 18(3), 188-196, 2005.

${ }^{6}$ Taylor, L.A. and Meek, T.T., "Microwave Processing of Lunar Soil," (AAS 03-716) Proc. ILC/JLEWG5 p109-125, (2003)

${ }^{7}$ Lunar Regolith Simulant Workshop, Huntsville, AL, 2004

${ }^{8}$ Ethridge, E.C. and W. Kaukler, "Microwave Processing of Planetary Surfaces for the Extraction of Volatiles," 49th AIAA Aerospace Sciences meeting, Orlando, FL January 2-8, (2011).

${ }^{9}$ McCoustra, M.R.S. and D.A. Williams, "Physiochemical Processes on Surfaces: The Gas Dust Interaction in Instellar Molecular Clouds," Final Report on PPARC Research Grant GR/L57562 (2001).

${ }^{10}$ Cooper, J.R. and R.B. Dooley, "Revised Release on the Pressure Along the Melting and Sublimation Curves of Ordinary Water Substance," Inter. Assoc. Prop. Water and Steam (2008).

${ }^{11}$ Heiken, G.H., D.T. Vaniman, and B.M. French, Lunar Sourcebook, Lunar and Planetary Institute, (1991).

${ }^{12}$ Crews, C., "Thermophysical Properties of Lunar Media: Part II," in Advances in Heat Transfer, Vol. 10, J.P. Hartnett and T. F. Irvine, eds. Academic Press, NY, (1974). 


\section{Finite Element Analysis of Three Methods for Microwave Heating of Planetary Surfaces.}

Edwin Ethridge, PhD

Materials and Processes Laboratory

NASA Marshall Space Flight Center

Huntsville, AL 35812

William Kaukler, PhD

Assistant Research Professor in Chemistry

U. of Alabama - Huntsville, AL 35899

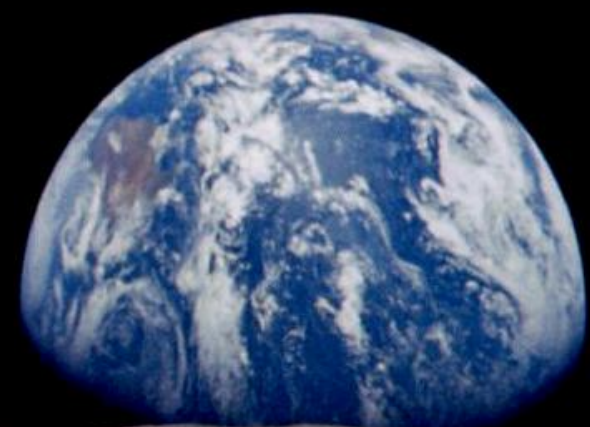

50't Aerospace Sciences Meeting Nashville, TN

9 to 12 January, 2012 


\section{Microwave Heating of Regolith}

- Sintering of regolith for construction

- Melting of regolith (perhaps more suitable for solar heating)

Fibers - cables, insulation

Foams - insulation

Dust mitigation - landing sites

- Volatiles, in particular water extraction - potentially the most important application

Water (and oxygen) for habitation

Water for radiation protection (extended missions)

Propellant production

Hydrogen for nuclear thermal and electric propulsion BEO

Oxygen and Hydrogen for near Earth propulsion 


\section{Advantages of Microwaves for the Extraction of Lunar Water}

- Lunar regolith (in vacuum) has a very low thermal conductivity

It is an Insulator very low heat flow.

- Microwave energy penetrates the regolith heating from the inside out

Penetration depth is dependent on Frequency.

- Heating regolith sublimes water ice, sublimation is a simple phase change, not a chemical reduction reaction.

- Conversion from electricity through microwaves to regolith heating, $>50 \%$ efficient, only heat regolith and ice.

Rapid heating and instantaneous response,

- Excavation may not be required,

Cryogenic water is as hard as granite

Little if any disruption of lunar dust

Less Earth launch mass, less power, less complexity,

less infrastructure 


\section{Proof of Principle Water Extraction}
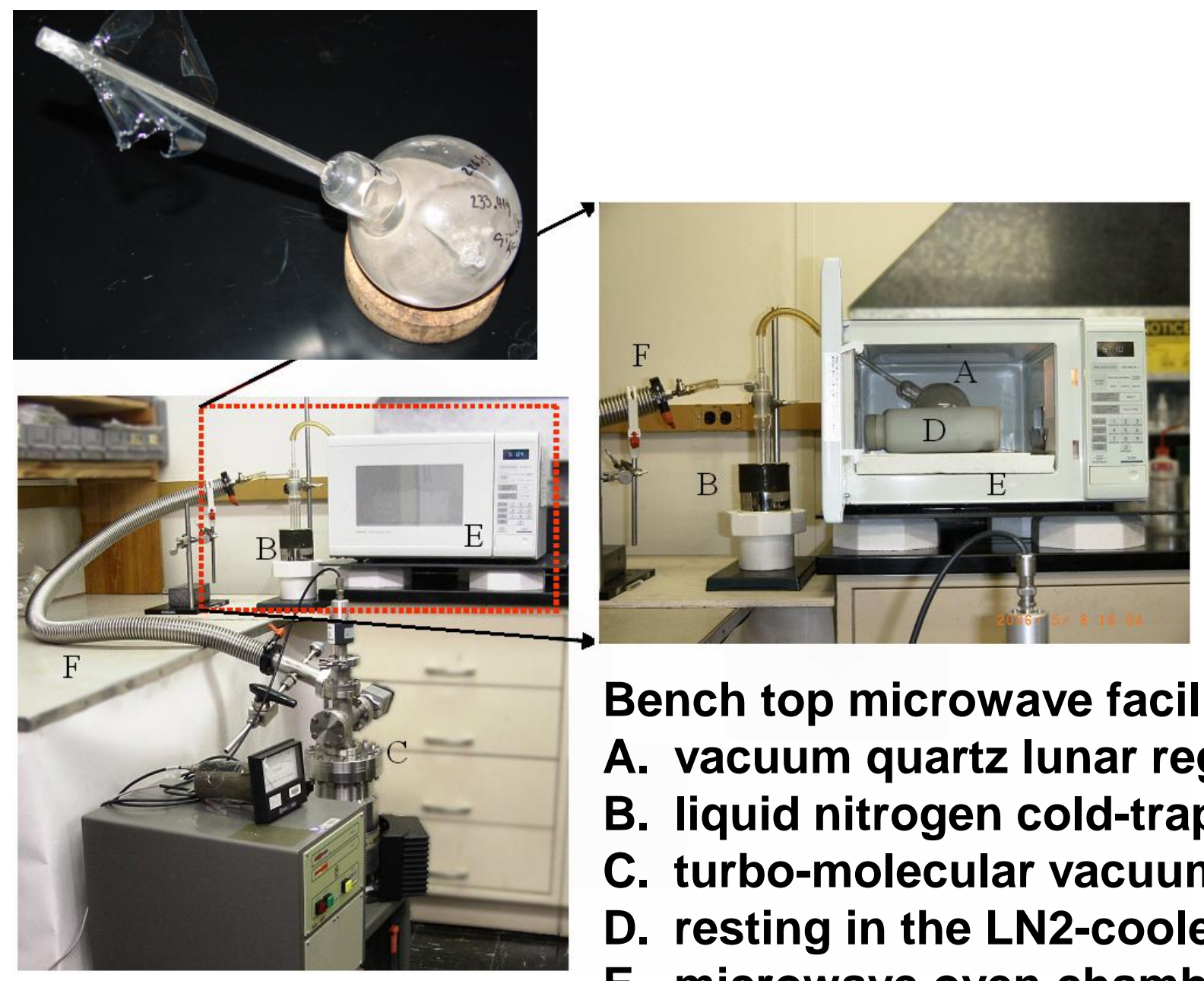

Bench top microwave facility

A. vacuum quartz lunar regolith simulant vessel

B. liquid nitrogen cold-trap

C. turbo-molecular vacuum pump

D. resting in the LN2-cooled regolith bed

E. microwave oven chamber 


\section{Proof of Principle Results}

- Microwaves (2.45 GHz) couple with and heat regolith simulant (JSC-1A) at cryogenic temperatures,

- Water ice rapidly sublimes $<0^{\circ} \mathrm{C}$.

- Water ice efficiently recaptured on a external cold trap 


\section{Demonstration Experiments with a Microwave Apparatus on a Rover}

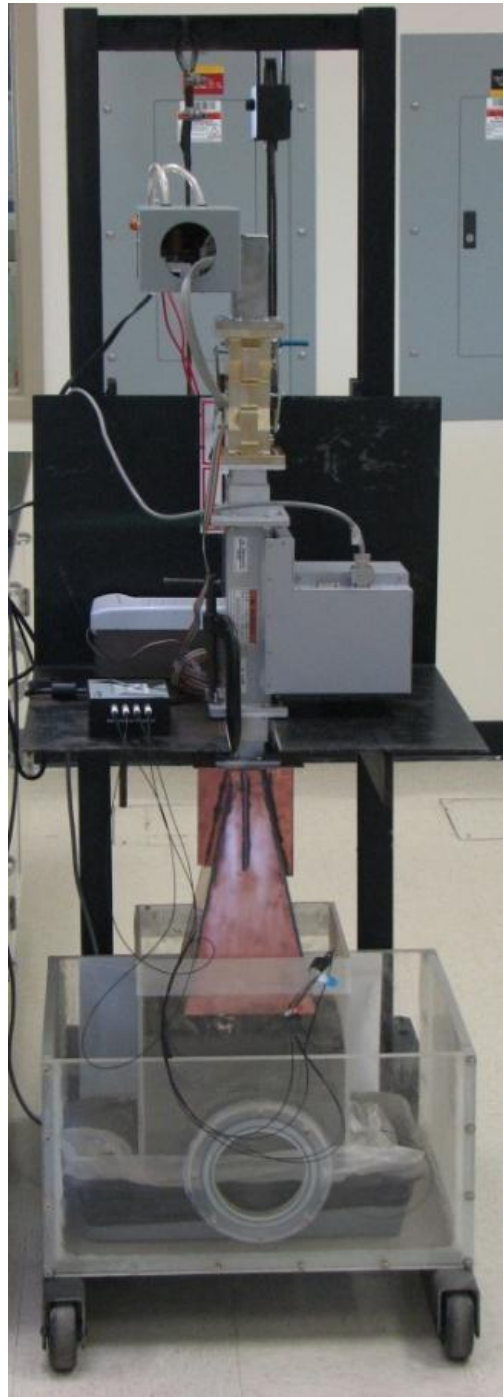

Beamed microwaves $(2.45 \mathrm{GHz}, 1000 \mathrm{~W})$ through an isolator and auto-tuner into a homemade high gain copper horn into lunar regolith simulant JSC-1A.

Measured temperatures with a Luxtron fluoro-optic fiber optic sensors (4 each).

Temperature increase is consistent with Beer Lambert law attenuation. 


\section{Dielectric Properties}

- Dielectric Properties are a function of frequency.

- Properties are also a function of temperature.

- Microwave penetration depth depends on the regolith properties.

- The measurement of the complex magnetic permeability is required to evaluate microwave penetration and heating.

- Properties of lunar regolith simulants were not known, so three years ago we setup to make the measurements. 


\section{Dielectric Property Measurements Lunar Regolith Simulant - JSC-1A}

NASA

- MSFC has a Microwave Characterization Laboratory for Dielectric Measurements

- Developed methods for measuring dielectric properties by two methods:

Transmission in waveguide Resonant cavity

- Measured the complex electric permittivity; $\varepsilon^{\prime}-\varepsilon^{\prime \prime} j$ $10 \mathrm{GHz}$ from cryogenic to room temperature
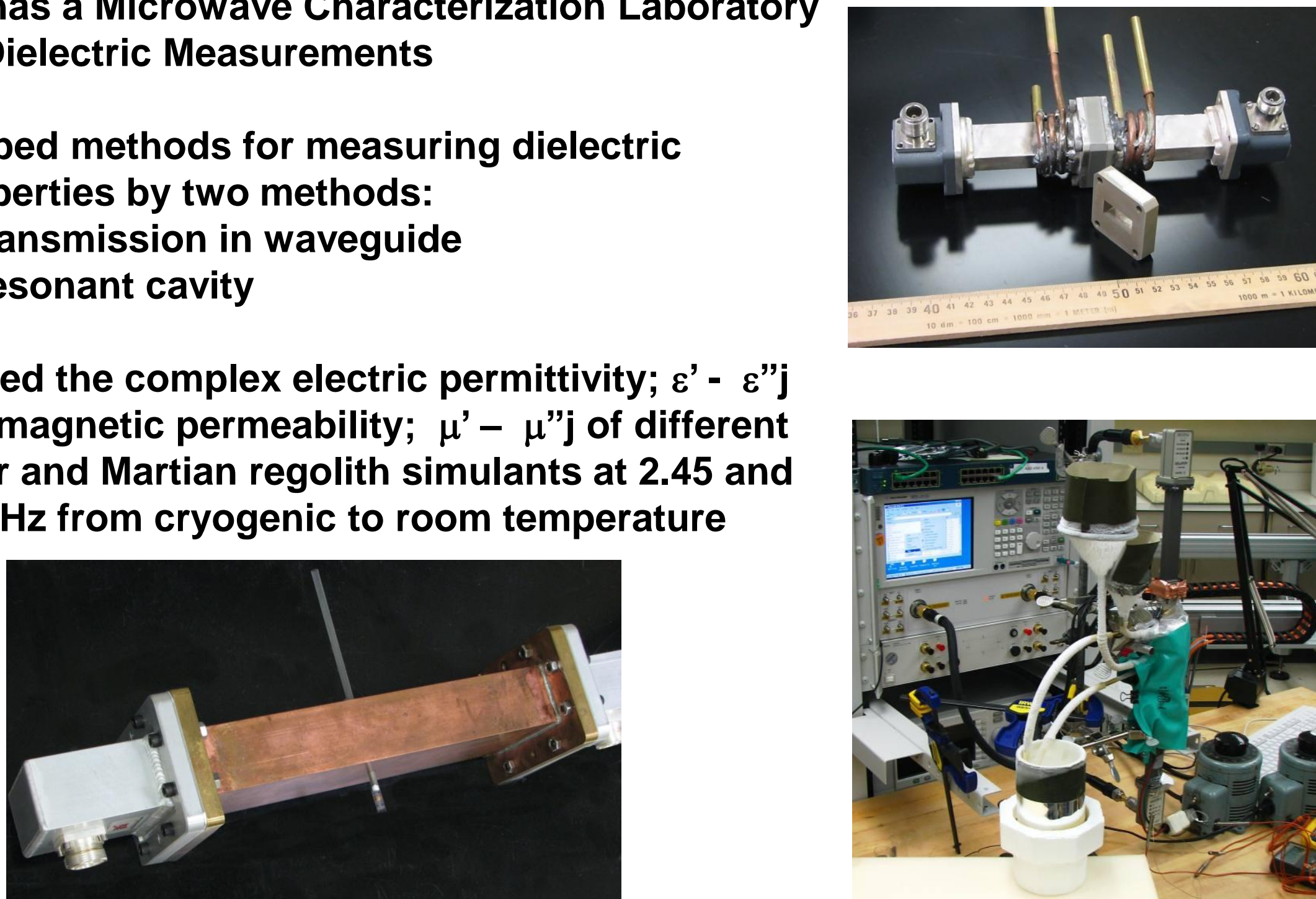
and magnetic permeability; $\mu$ ' $-\mu$ "j of different lunar and Martian regolith simulants at 2.45 and




\section{Conclusions from Dielectric Property Measurements}

- Permittivity and Permeability are a slight function of frequency. Roughly a $10 \%$ increase from 0.9 to $10 \mathrm{GHz}$.

- Permittivity and Permeability are a slight function of temperature. Roughly a $10 \%$ increase from -100 to $+100^{\circ} \mathrm{C}$.

- The Permittivity and Permeability of JSC-1A and modifications with iron additions are with in $5 \%$ of JSC-1A (at $2.45 \mathrm{GHz}, \varepsilon \sim 4-0.1 \mathrm{j}, \mu \sim 1-0.01 \mathrm{j}$ )

- The dielectric constant $\left(\varepsilon^{*}=3.8\right)$ of Apollo regolith* is only slightly different from JSC-1A at room temperature.

- Thermal runaway is known to occur when regolith is heated above $200^{\circ} \mathrm{C}$. There could be significant differences between JSC-1A and Apollo regolith (containing nano-Fe) at higher temperatures. 


\section{Application of COMSOL Multiphysics FEM}

NASA

- Processing parameters and hardware requirements for microwave water extraction is a complex multiphysics problem.

- Microwave coupling to materials and heating is dependent on frequency and materials properties.

- Materials properties are a function of frequency and temperature.

- Can calculate microwave penetration and heating, with frequency and temperature dependent lunar soil dielectric properties.

- Can model sublimation and water vapor percolation through the soil (porous media) characterized by the Darcy constant.

- Parametric modeling will permit the evaluation of processing parameters most suitable for prototype hardware development, testing, and trade studies. 


\section{COMSOL FEM Multiphysics Parametric Analysis of Microwave Heating Models}

Three microwave heating models examined with COMSOL Multiphysics

- Microwave Oven (2.45 GHz)

- Microwave Horn (2.45 , 24.5, and 75 GHz)

- Microwave Probe down borehole (2.45 GHz)

a. Monopole launcher

b. Unidirectional launcher 


\section{COMSOL V4.2a Model Builder Physics - Microwave Heating Study - Frequency Transient}

File Edit View Options Help

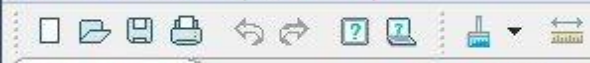

\section{Model Builder}

(3) 2OvenRegolithMHRegolithSTPconst300K500W10 min . $\mathrm{mph}$,

E Global Definitions

(1) Model $1(\bmod 1)$

$\equiv$ Definitions

A Geometry 1

(s) Materials

Microwave Heating $(\mathrm{mh})$

D Microwave Heating Model 1

D Electromagnetic Heat Source 1

Dat Boundary Electromagnetic Heat Source 1

Det Perfect Electric Conductor 1

Det Thermal Insulation 1

D Initial Values 1

$@$ Wave Equation, Electric 1

(at) Port 1

Impedance Boundary Condition 1

(D) Perfect Magnetic Conductor 1

(7) Mesh 1

Study 1

Step 1: Frequency-Transient

$p_{p}$, Solver Configurations

\section{韧 Solver 1}

$\frac{\partial u}{\partial t} f$ Compile Equations: Frequency-Transient

u,v,w Dependent Variables 1

[x. Time-Dependent Solver 1

Direct

Advanced
Segregated 1

$\triangle$ Iterative1

$\mathbb{Q}$ Incomplete LU

Multigrid 1
(9) Presmoother
(8) Postsmoother
(3) Coarse Solver

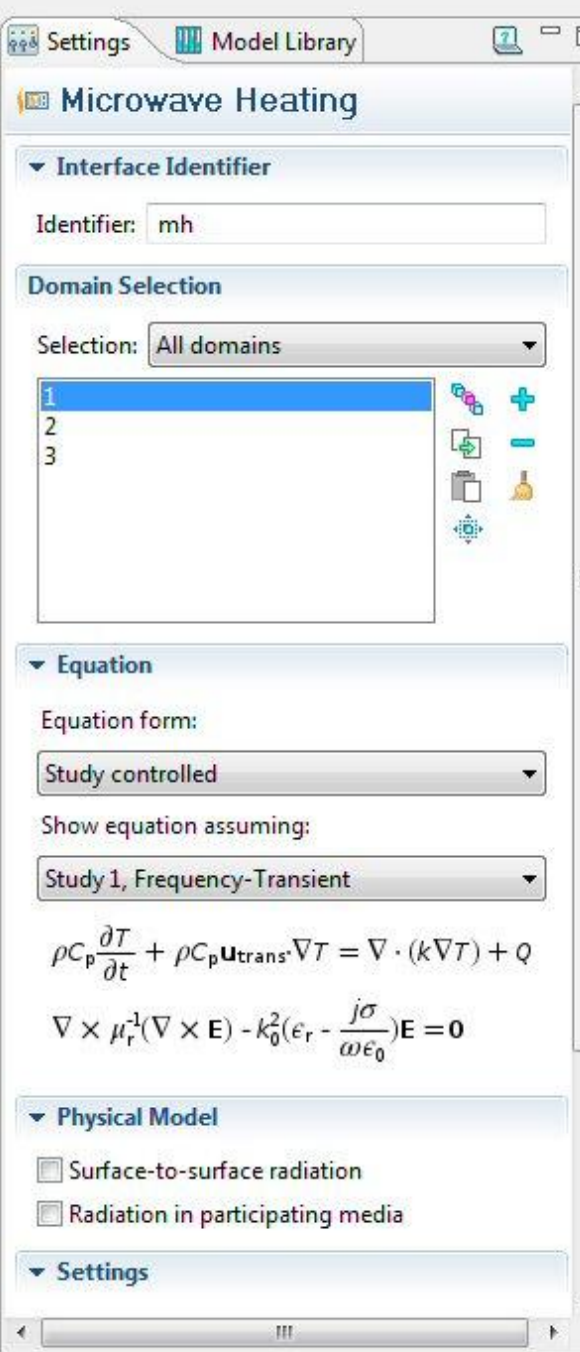

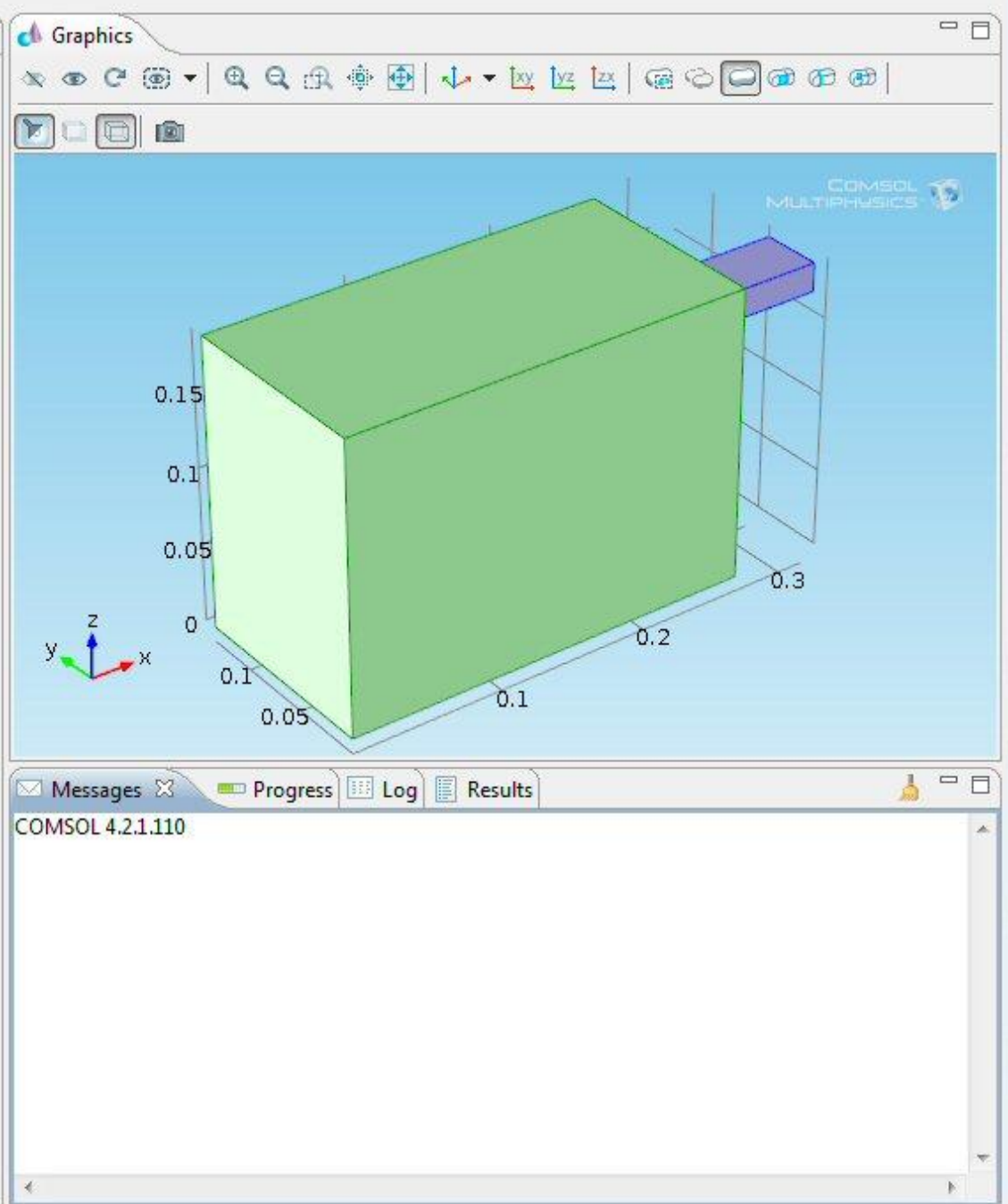

$261 \mathrm{MB} \mid 288 \mathrm{MB}$ 


\section{Table I. Materials Values for "Room Temperature" Calculations}

- Thermal Conductivity k (dry sand) $0.35 \mathrm{~W} / \mathrm{m}^{\star} \mathrm{K}$

- Specific Heat

$\mathrm{Cp}$

c6

- Density

$\rho$ measured

- Electrical conductivity

S

$0.85 \mathrm{KJ} / \mathrm{Kg}^{*} \mathrm{~K}$

- Permittivity

$\varepsilon^{\prime}-\varepsilon^{\prime \prime} \mathrm{j}$

$1.66 \mathrm{~g} / \mathrm{cm}^{3}$

- Permeability

$\mu^{\prime}-\mu^{\prime \prime} \mathrm{j}$

$4-0.1 j$

$1-0.01 j$

\section{- Material Contents}

\begin{tabular}{|llllll|}
\hline Property & Name & Value & Unit & Property group \\
\hline$\checkmark$ Heat capacity at constant $\mathrm{pr} \ldots$ & $\mathrm{Cp}$ & $850[\mathrm{~J} / \ldots$ & $\mathrm{J} /(\mathrm{kg} \ldots$ & Basic \\
$\checkmark$ Density & rho & $1.66[\mathrm{~g} / \ldots$ & $\mathrm{kg} / \ldots$ & Basic \\
$\checkmark$ Electrical conductivity & sigma & 0 & $\mathrm{~S} / \mathrm{m}$ & Basic \\
$\checkmark$ Relative permittivity & epsil... & $4-0.1^{\star} \mathrm{j}$ & 1 & Basic \\
$\checkmark$ Relative permeability & mur & $1-0.01^{\star} \mathrm{j}$ & 1 & Basic \\
$\checkmark$ Thermal conductivity & $\mathrm{k}$ & 0.35 & $\mathrm{~W} /(\ldots$ & Basic \\
\hline
\end{tabular}




\section{COMSOL Meshing 3-D Geometry}

- Mesh statistics

Property Value

- Minimum element quality 0.09707

- Average element quality

0.7269

- Tetrahedral elements

4582

- Triangular elements

1072

- Edge elements

170

- Vertex elements

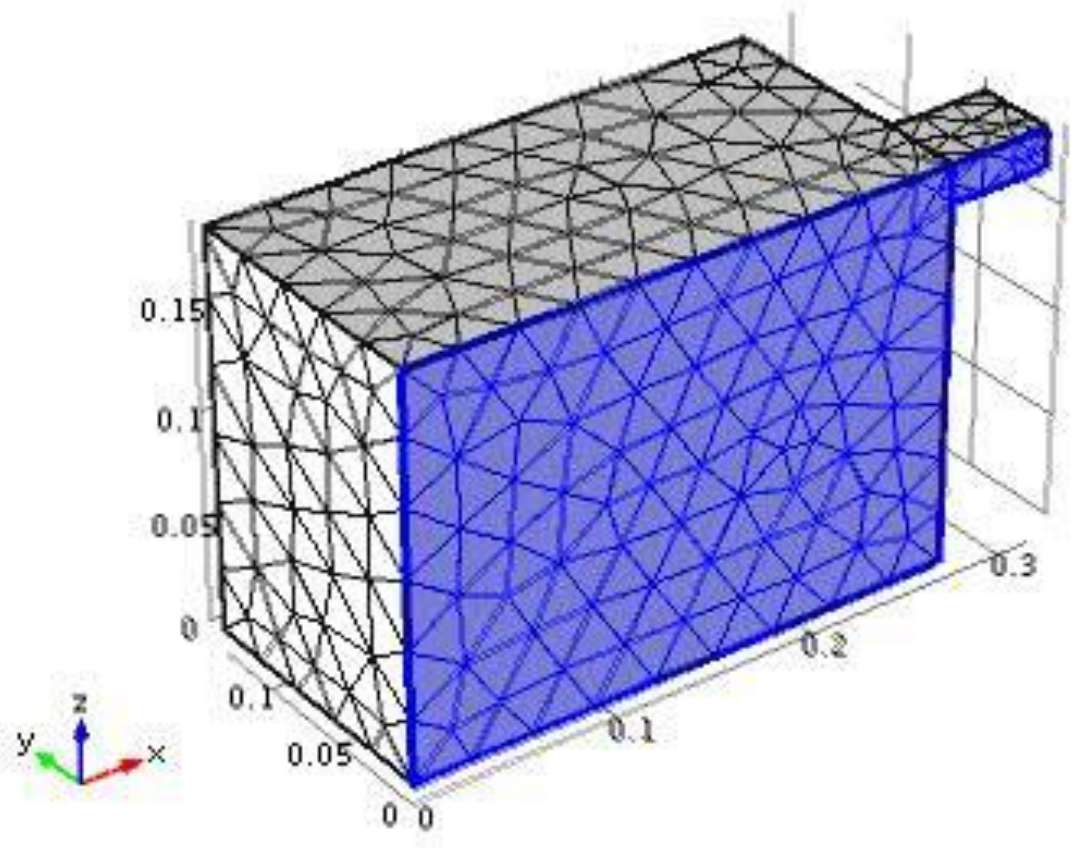




\section{Slices showing Electric Energy Density Through the Microwave Oven}

- Planar slices through the microwave oven

- Electric energy density time average (1E-13 to $1 \mathrm{E}-12 \mathrm{~J} / \mathrm{m}^{\wedge} 3$ )

- Through the oven and within the cylindrical sample.

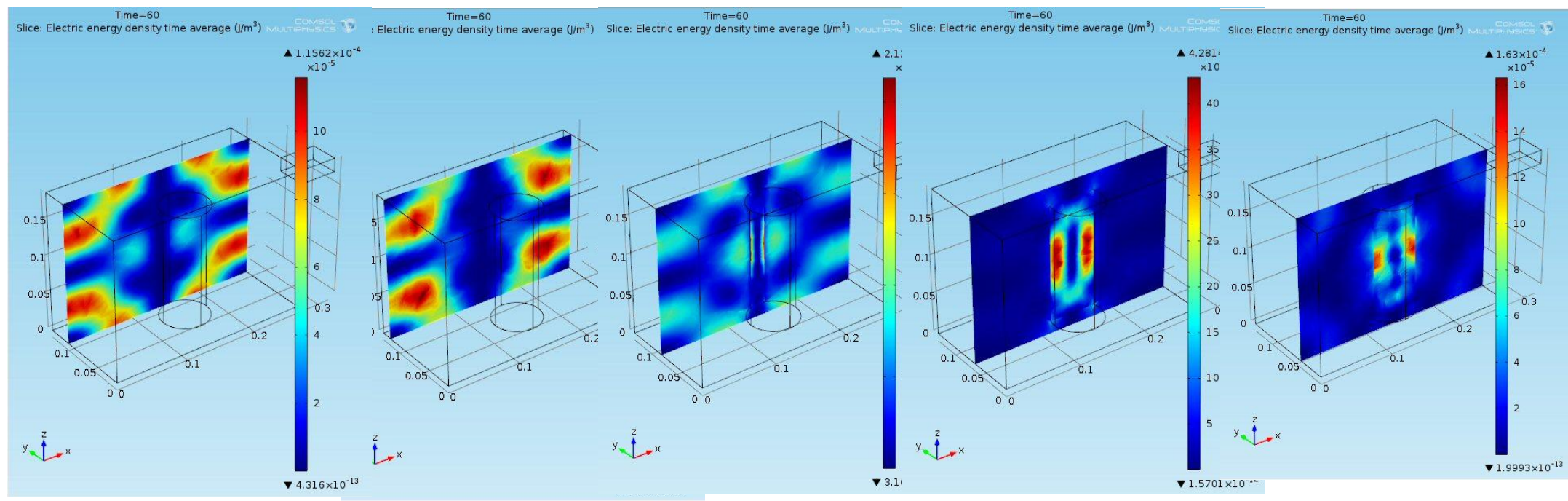




\section{Effect of the Dielectric Properties on Microwave Heating (2.45 GHz, 5 min, 500 W)}

$\varepsilon=3-0.01 \mathrm{j} \mu=1-0.01 \mathrm{j} \quad 303$ to $367 \mathrm{~K}$

Time $=10 \mathrm{~min}$ IsoTemperature (K)

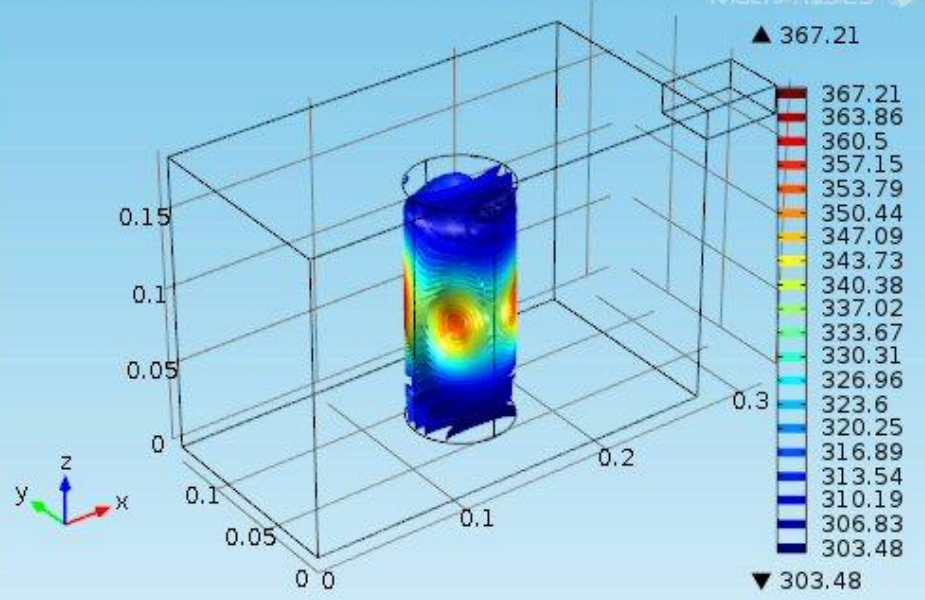

$\varepsilon=4-0.1 \mathrm{j} \mu=1-0.01 \mathrm{j} \quad 305$ to $396 \mathrm{~K}$

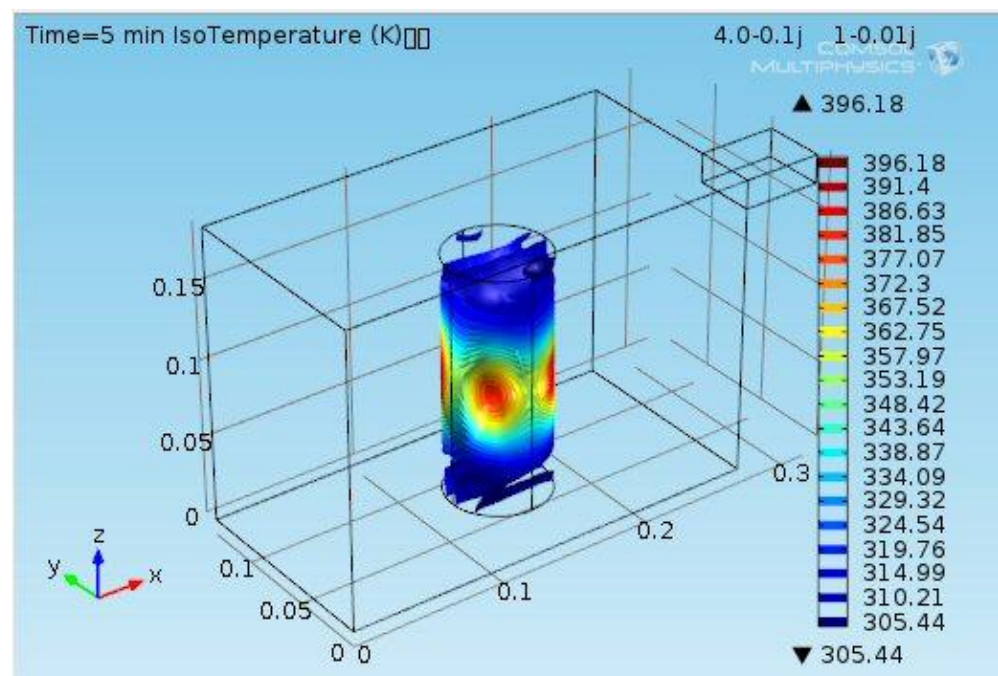

$\varepsilon=4.5-0.2 \mathrm{j} \mu=1-0.2 \mathrm{j} \quad 319$ to $383 \mathrm{~K}$

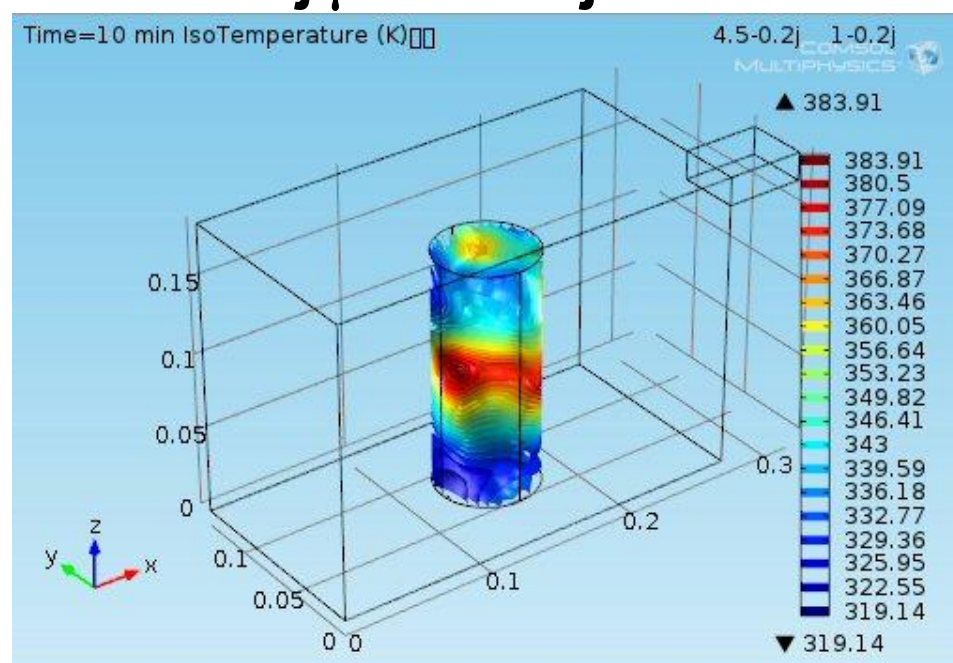

$\varepsilon=4.5-0.2 \mathrm{j} \mu=1.5-0.4 \mathrm{j} 316$ to $395 \mathrm{~K}$

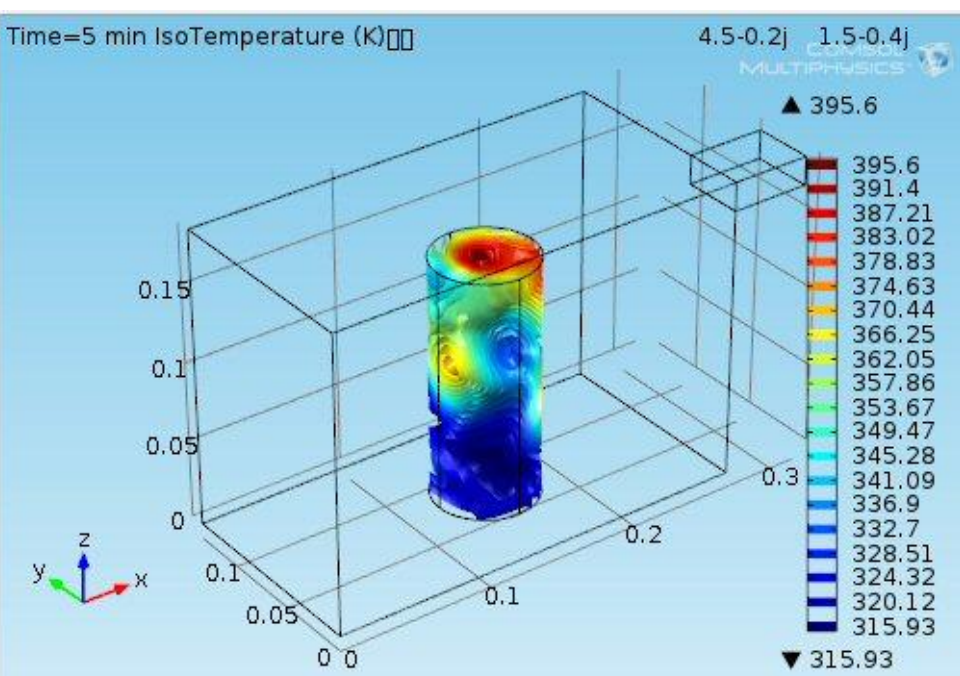




\section{Microwave oven (2.45 GHz) Heating}

- Cylinder of lunar regolith simulant, JSC-1A

- Starting at room temperature (300K)

- Isotherms
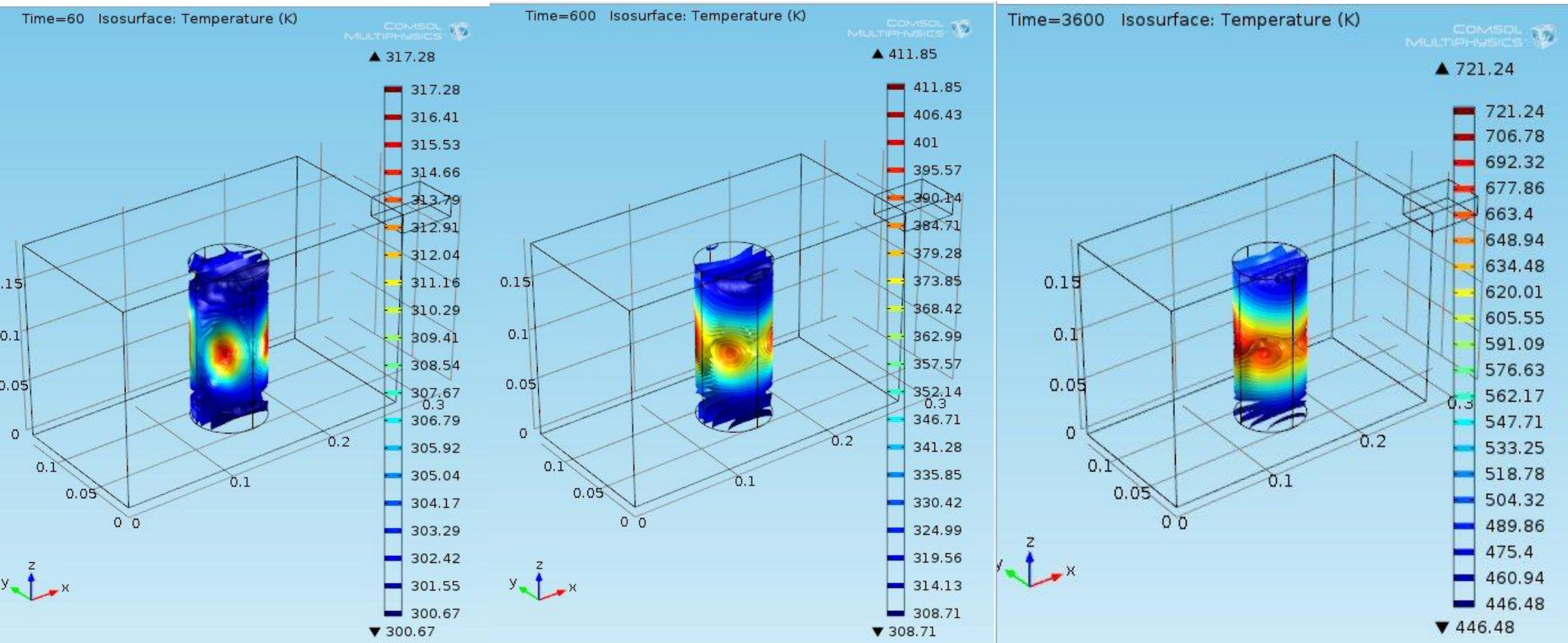


\section{Melting of JSC-1a Simulant}

- Conventional Microwave Oven in air

- Thermal Runaway Heating 5 minutes, aluminia crucible

- No native iron was present.

- Bubbles from trapped air in the powder

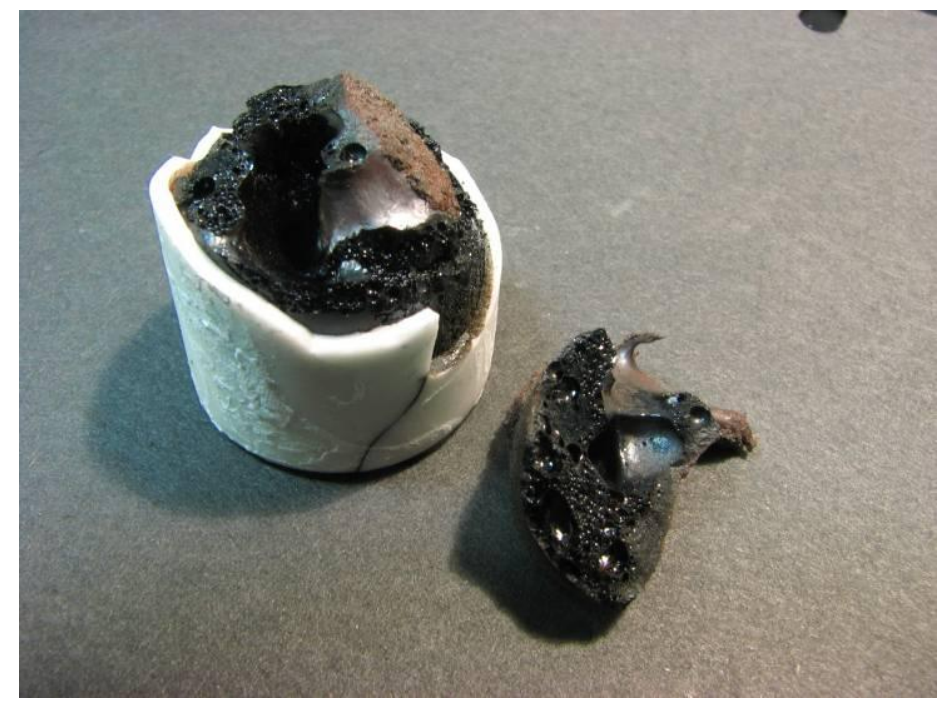




\section{Table II. Materials Properties for Calculations Initial Temp $100^{\circ} \mathrm{K}$}

- Thermal Conductivity k

- Specific Heat

- Density measured

- Electrical conductivity

- Permittivity*

- Permeability*

$$
\begin{array}{cc}
\varepsilon^{\prime}-\varepsilon^{\prime \prime}{ }^{*} & 4-0.1 \mathrm{j} \\
\mu^{\prime}-\mu^{\prime \prime} \text { j } & 1-0.01 \mathrm{j}
\end{array}
$$

Assumed constant temperature (100K to $400 \mathrm{~K}$ ) 


\section{Large Cylinder vs. Small cylinder NASA}

Diameter $6 \mathrm{~cm}$ Height $15 \mathrm{~cm}$

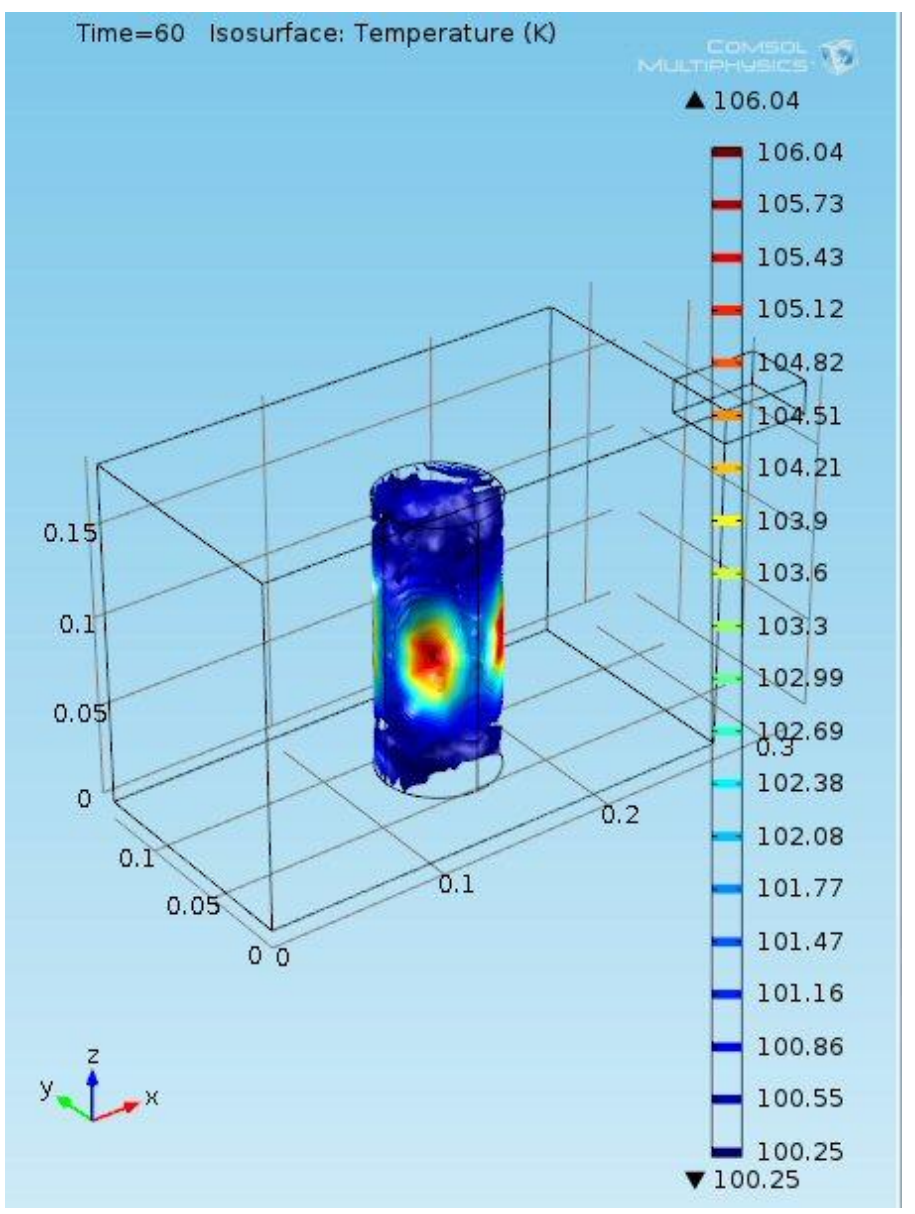

Radius $1 \mathrm{~cm}$

Height $1 \mathrm{~cm}$

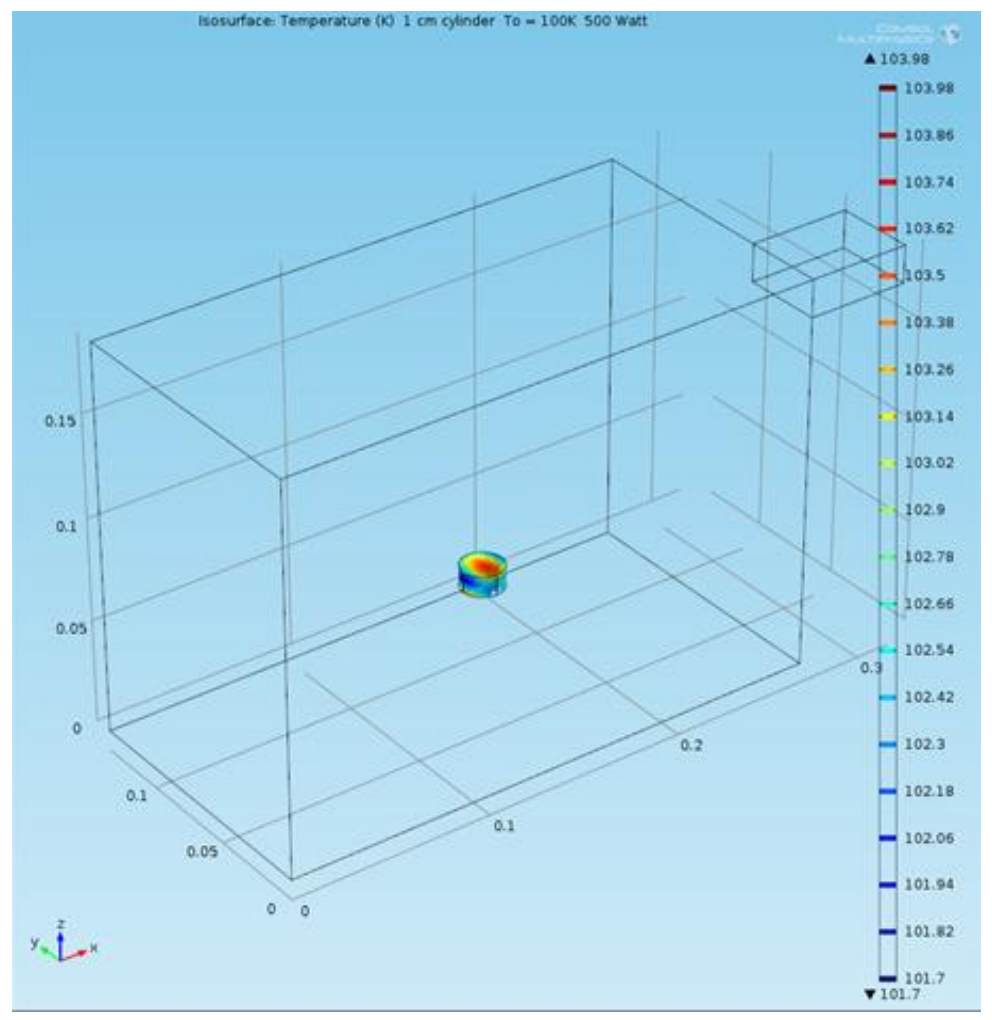




\section{Small cylindrical sample in Microwave oven $(2.45 \mathrm{GHz})$}

Plane slices showing the energy density in the oven.
Magnified view of the sample showing temperature isotherms in the sample. $50 \mathrm{~W}, 1 \mathrm{~min}, \mathrm{To}=100 \mathrm{~K}, \mathrm{~T}=102$ to $104 \mathrm{~K}$ Isosurface: Temperature (K) $1 \mathrm{~cm}$ cylinder To $=100 \mathrm{~K} 50$ Watt

$\Delta 103.98$

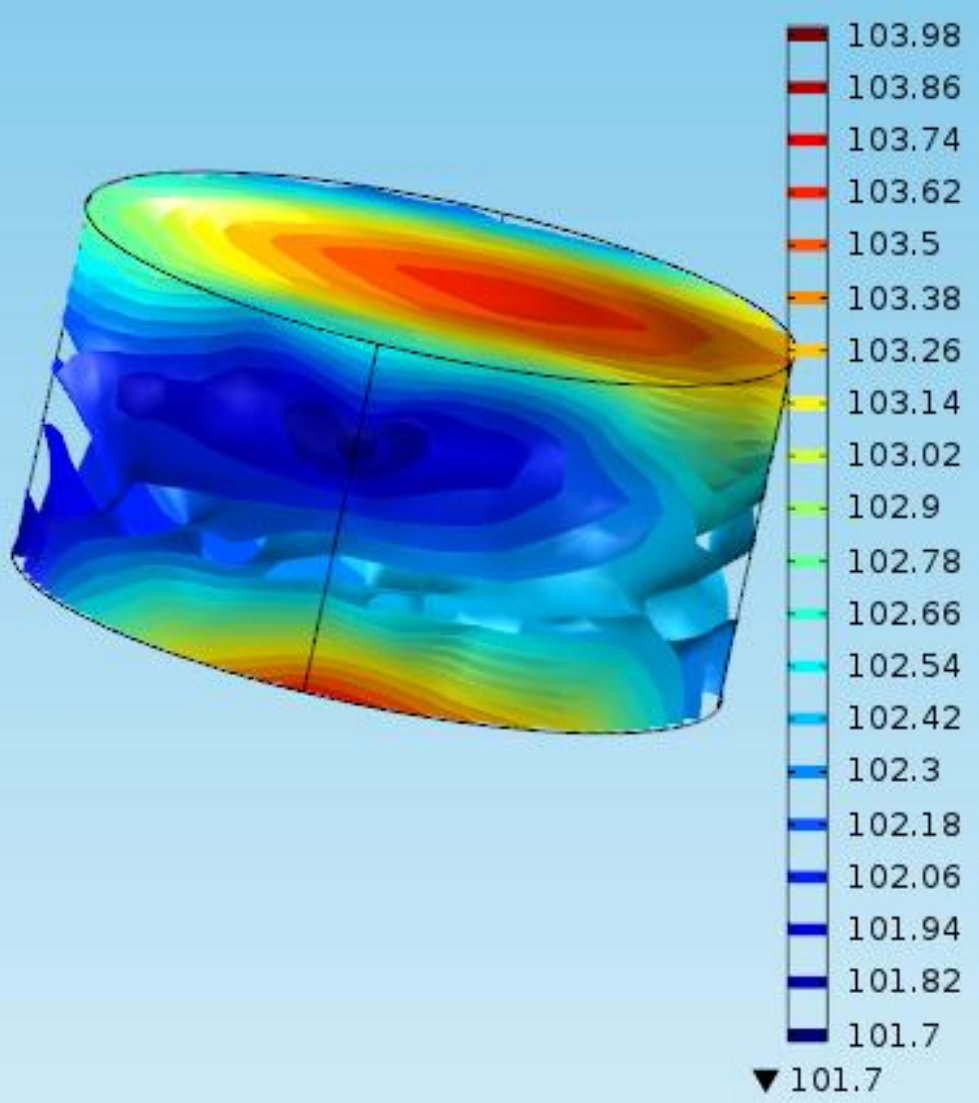

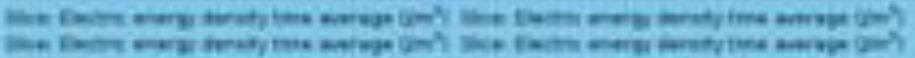

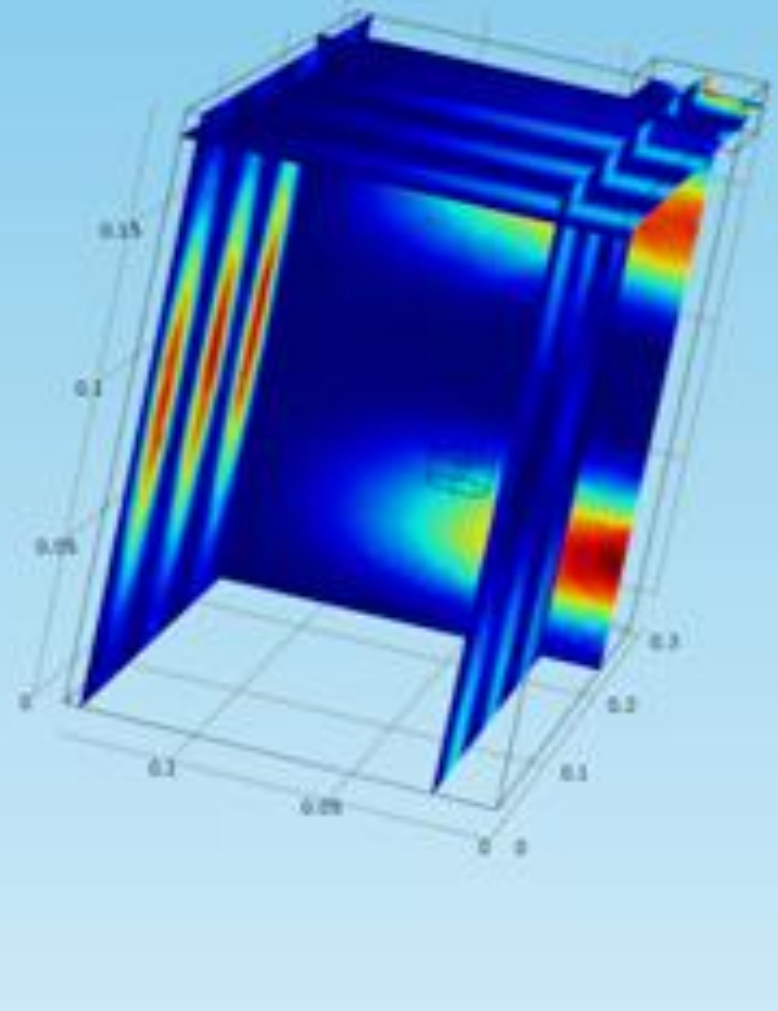

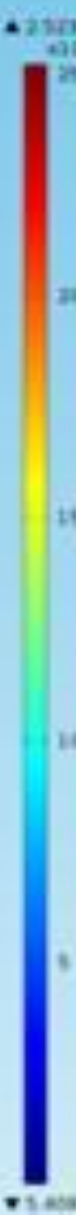




\section{Energy density within the microwave oven after moving the sample}

Plane slices showing the change in energy density in the oven after optimizing the sample position.
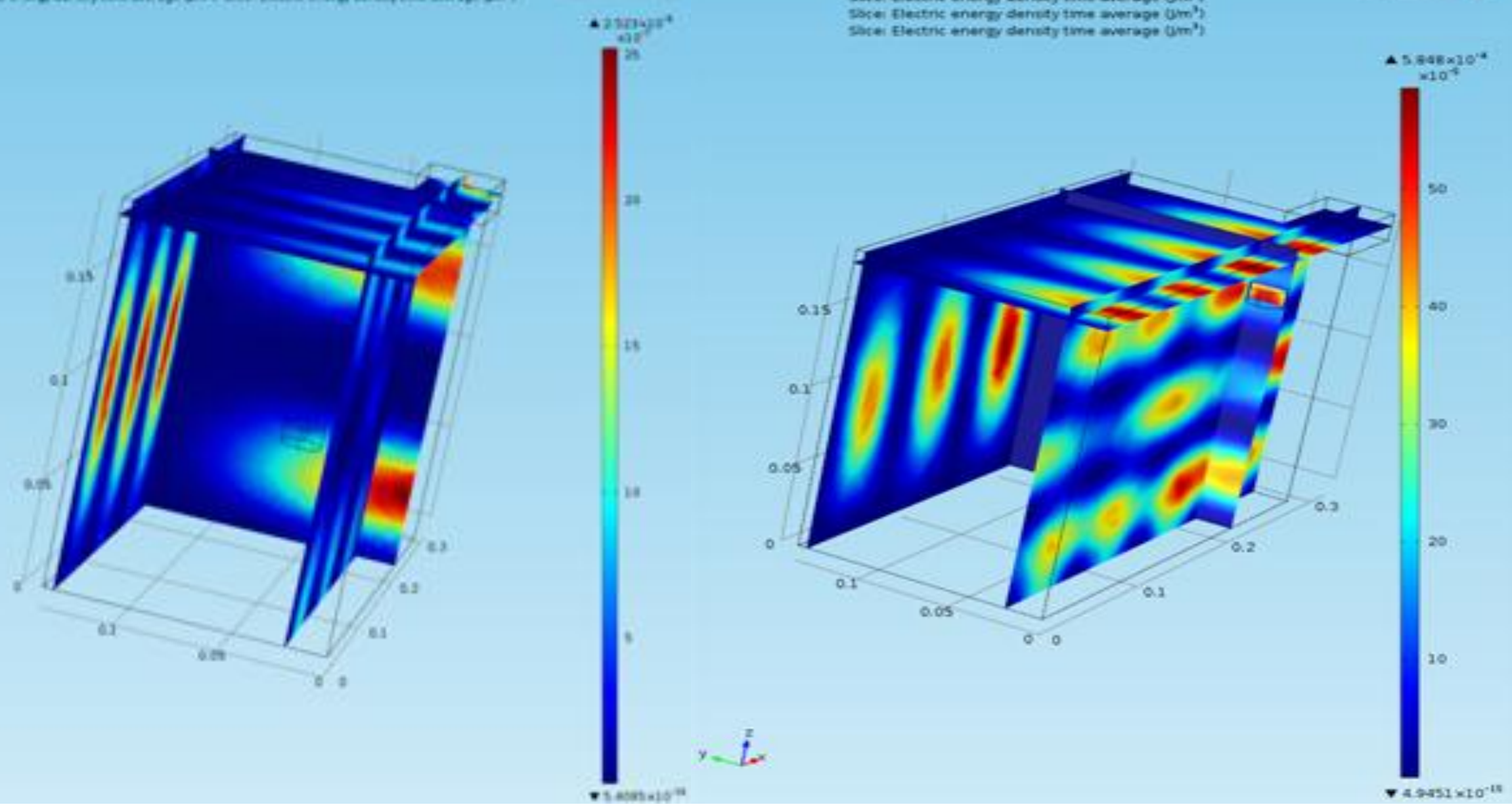


\section{Different Microwave Heating Models}

- Microwave Oven (2.45 GHz)

- Microwave Horn (2.45, 24.5 and 75 GHz)

- Microwave Probe down borehole (2.45 GHz)

a. Monopole launcher

b. Unidirectional launcher 


\section{Microwave Heating with a High Gain Horn}

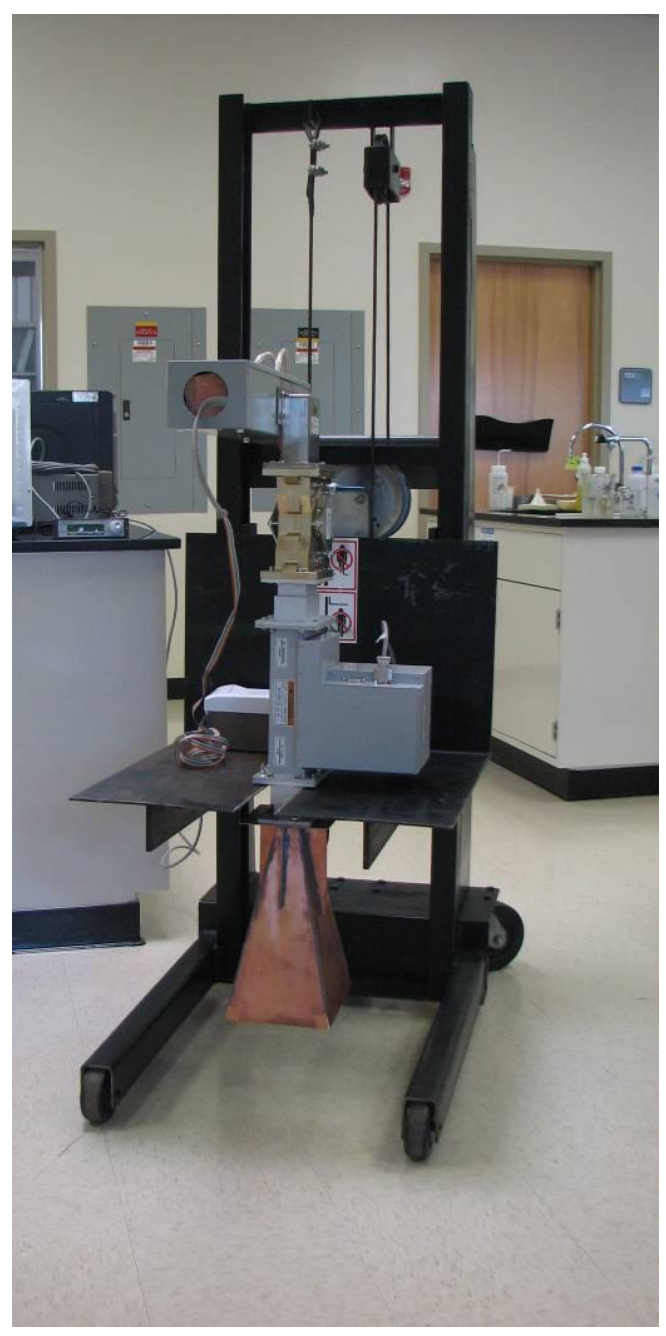

Magnetron source (2.45 GHz, 1000 W) with isolator, auto-tuner and copper high-gain pyramidal horn. 


\section{High Gain Microwave Horn $2.45 \mathrm{GHz}$}

Power of $\mathbf{5 0 0}$ watt, $\mathbf{1 0}$ minutes of heating. Plane slices showing the depth of penetration and heating into the surface (on the left).

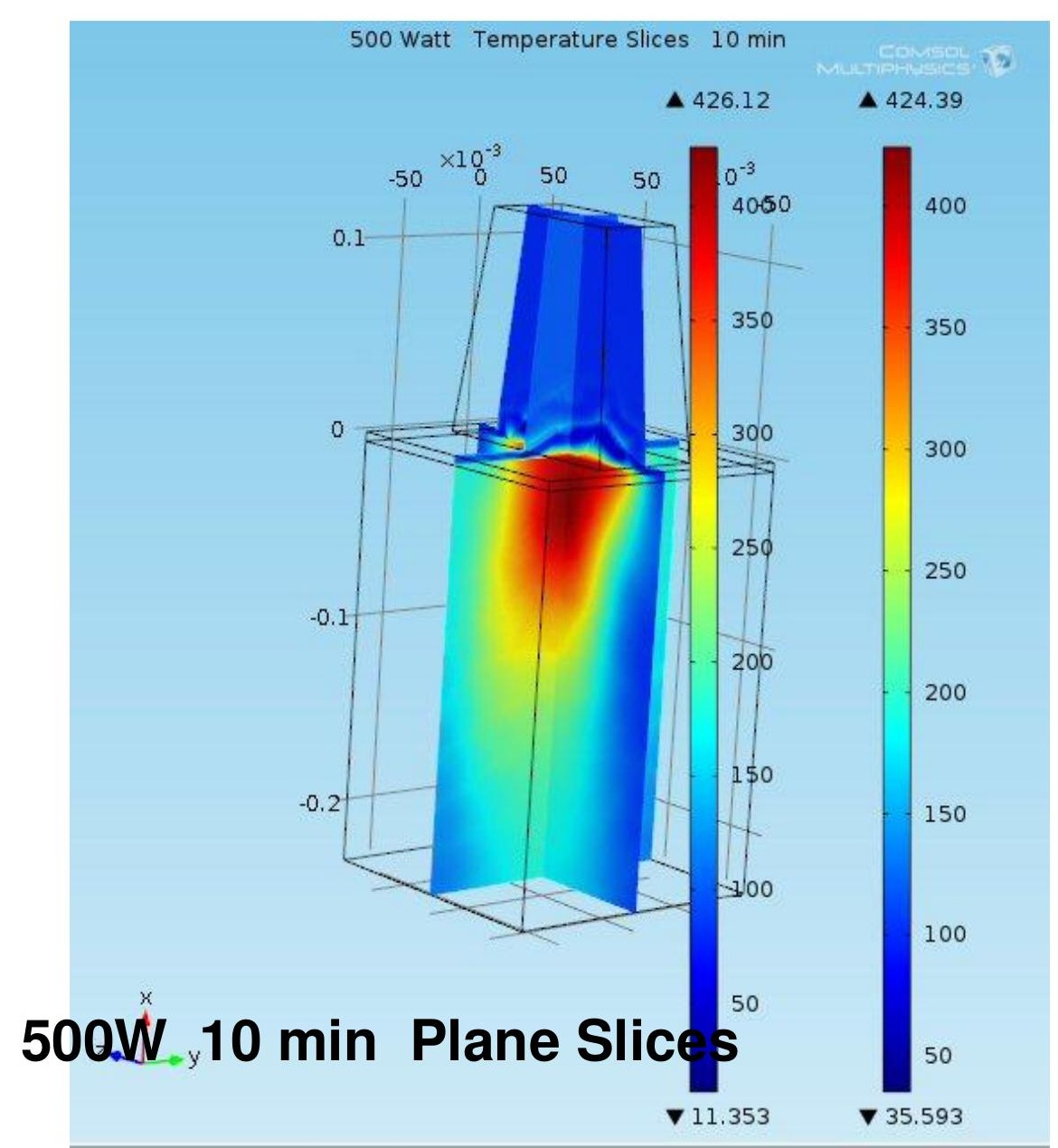

Isotherm at $200 \mathrm{~K}$ illustrating the volume heated sufficiently to completely volatilize the water ice present in the regolith.

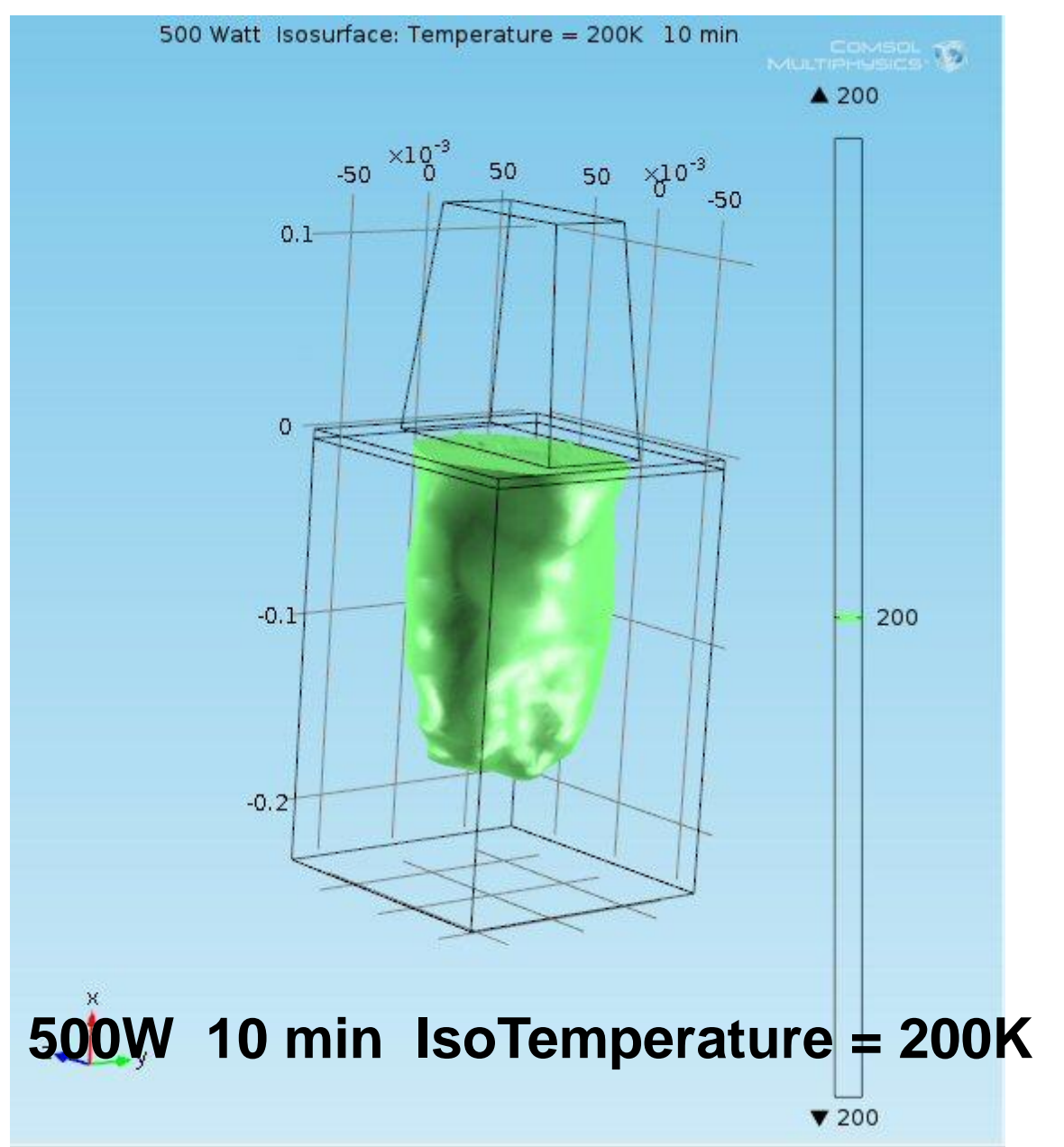




\section{Planetary/Asteroid Volatiles science using $24.5 \mathrm{GHz}$ microwaves}

NASA

Microwave horn $(24.5 \mathrm{GHz}) 0.5 \mathrm{~cm}$ from a planetary surface $(1 \mathrm{~cm}$ cube volume) in vacuum heating for 1 hour. Calculated Isothermal bands of heating within the cube. Temperatures approaching 1500K. Volatiles desorb, sublime, or decompose to be detected with sensors on the spacecraft.
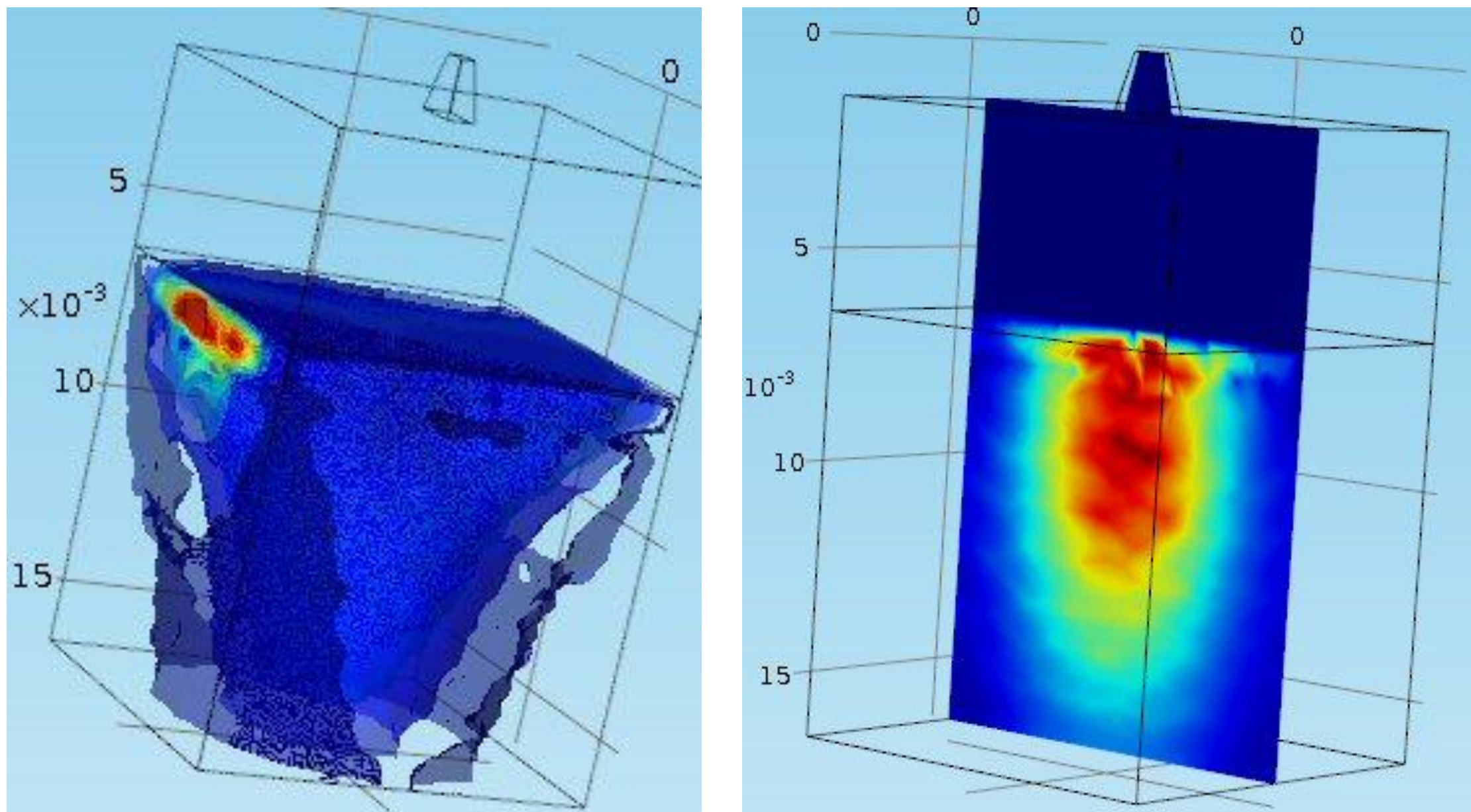


\section{K IsoTherm}

Isosurface Temperature $=\mathbf{2 0 0 K}$

According to the Water phase diagram, at 10-3 torr, water sublimation occurs within the isotherm

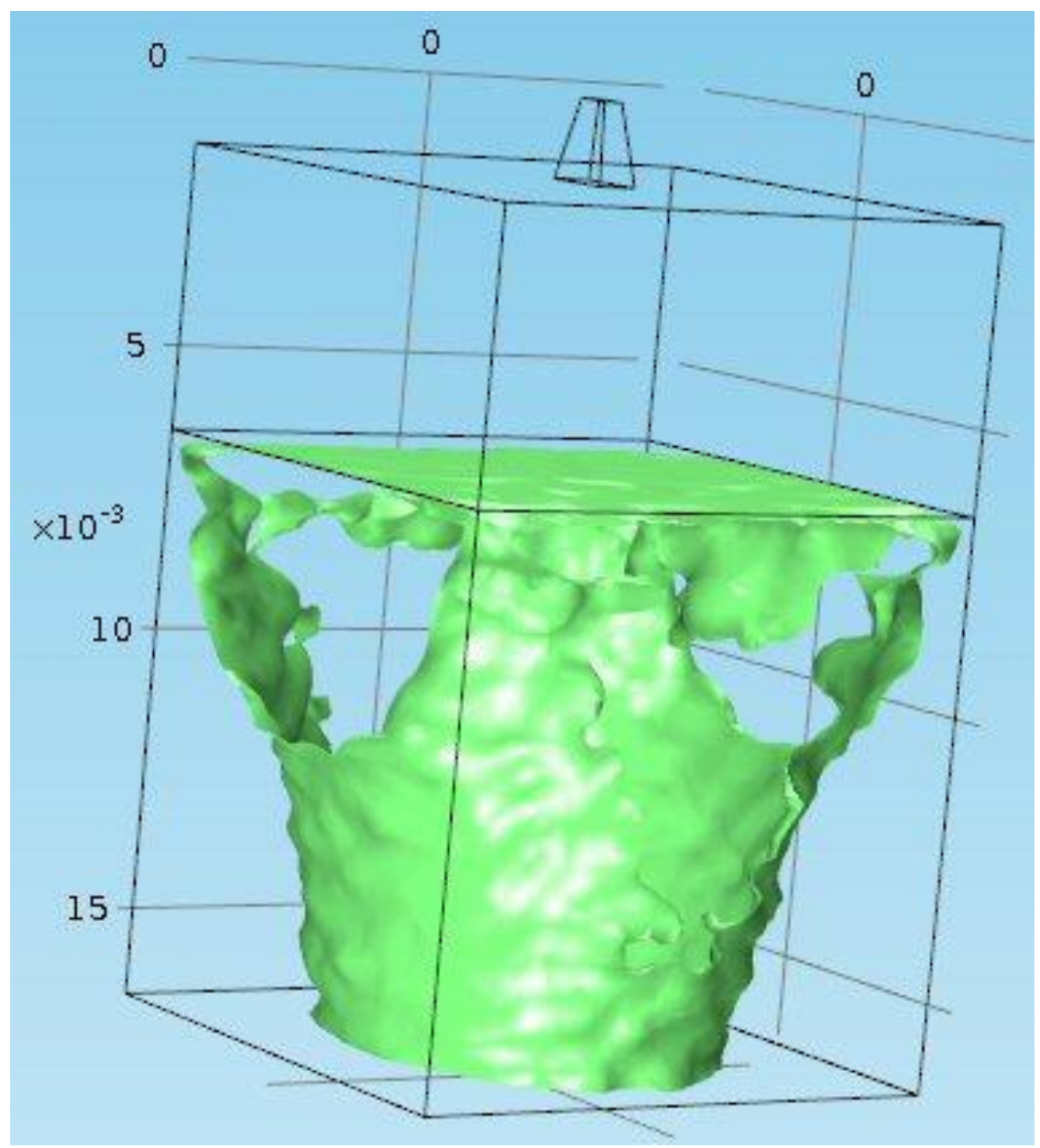




\section{Microwave Melting with High Energy 75 GHz}

Simplified Physics

High Power Density $3 \mathrm{KW} / \mathrm{cm}^{2}$

$75 \mathrm{GHz}$ for $2 \mathrm{sec}, 3 \mathrm{~cm}$ penetration

Temperatures $>$ melting temperature
Samples of lunar (JSC-1 and JS) and Martian (JSC-Mars1) regolith simulants melted with a $35 \mathrm{~kW}$ gyrotron device. The narrow microwave beam had a very high flux $\left(\sim 3 \mathrm{Kw} / \mathrm{cm}^{2}\right)$. Samples melted in seconds, $3 \mathrm{~cm}$ diameter, $0.5 \mathrm{~cm}$ thick.
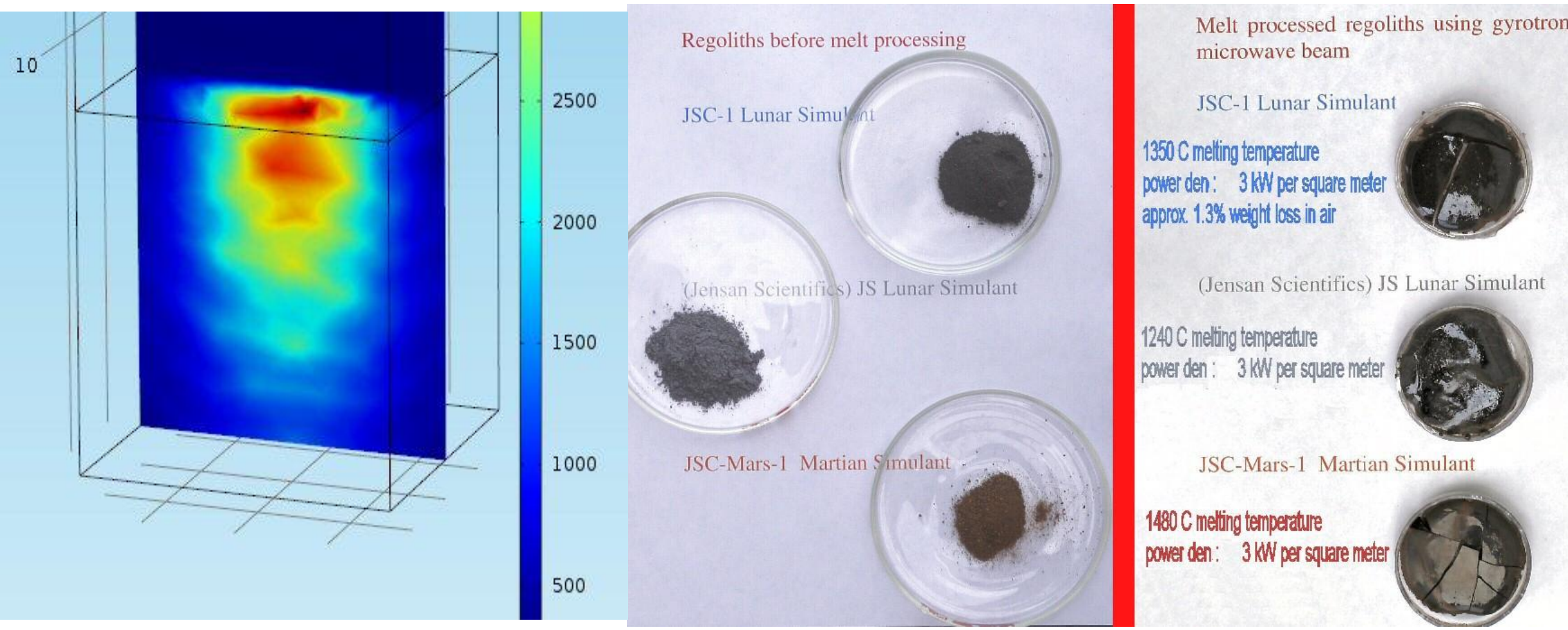

Melted by Gyrotron Technologies Inc. 


\section{Different Microwave Heating Models}

- Microwave Oven (2.45 GHz)

- Microwave Horn (2.45, 24.5 and 75 GHz)

- Microwave Probe down borehole (2.45 GHz)

a. Monopole launcher

b. Unidirectional launcher 


\section{Microwave Water Extraction From a Borehole}

- Vacuum chamber evaluation of the microwave water extraction down a $1 \mathrm{~m}$ borehole in lunar regolith simulant JSC-1A.
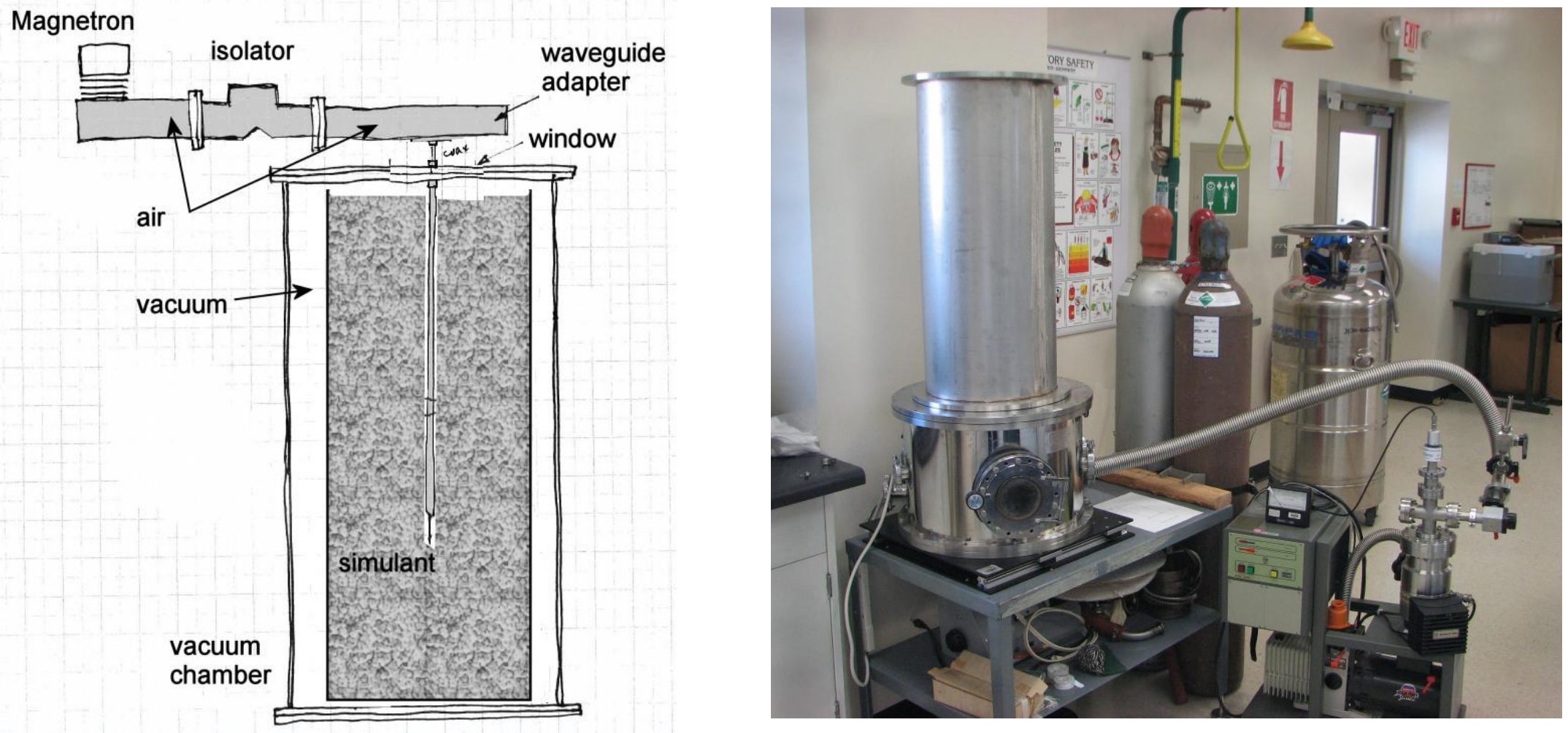


\section{Monopole Launcher Design}

Heating (1000 Watt 60 sec $2.45 \mathrm{GHz}$ ) with an improperly designed ground plane showing lobes along the coax feed line.
Correcting the launcher design, power radiates into and heats the regolith. Also using COMSOL to calculate S-Parameters and Smith Charts.
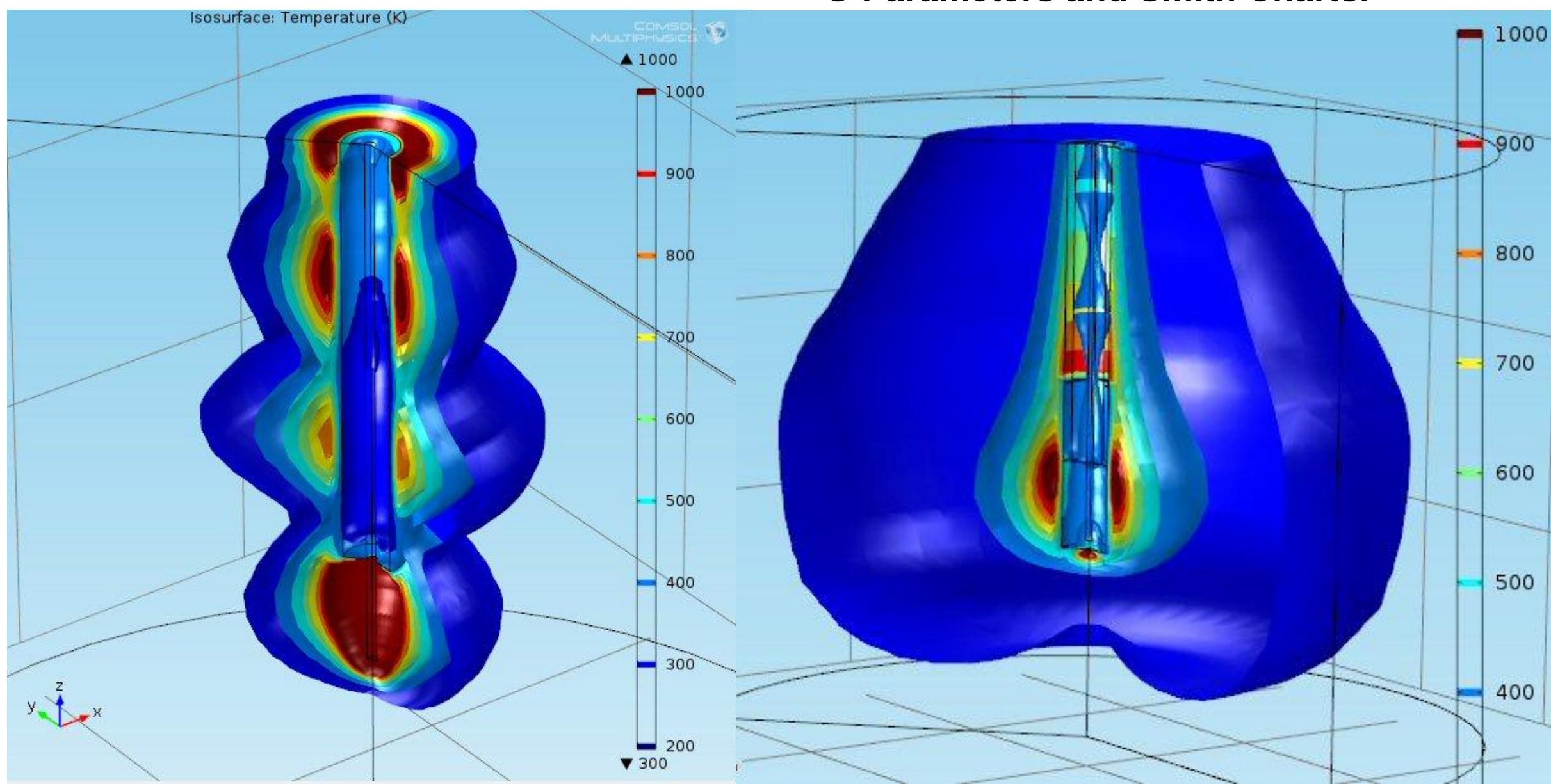


\section{Heating with Monopole Applicator}

1 minute of heating, $2.45 \mathrm{GHz} 100 \mathrm{~W}$ Isothermal plots from 100 to $200^{\circ} \mathrm{K}$ Brown region is $>200^{\circ} \mathrm{K}$
10 heating minutes

Region $>10 \mathrm{~cm}$ sufficiently heated to be devoid of water.

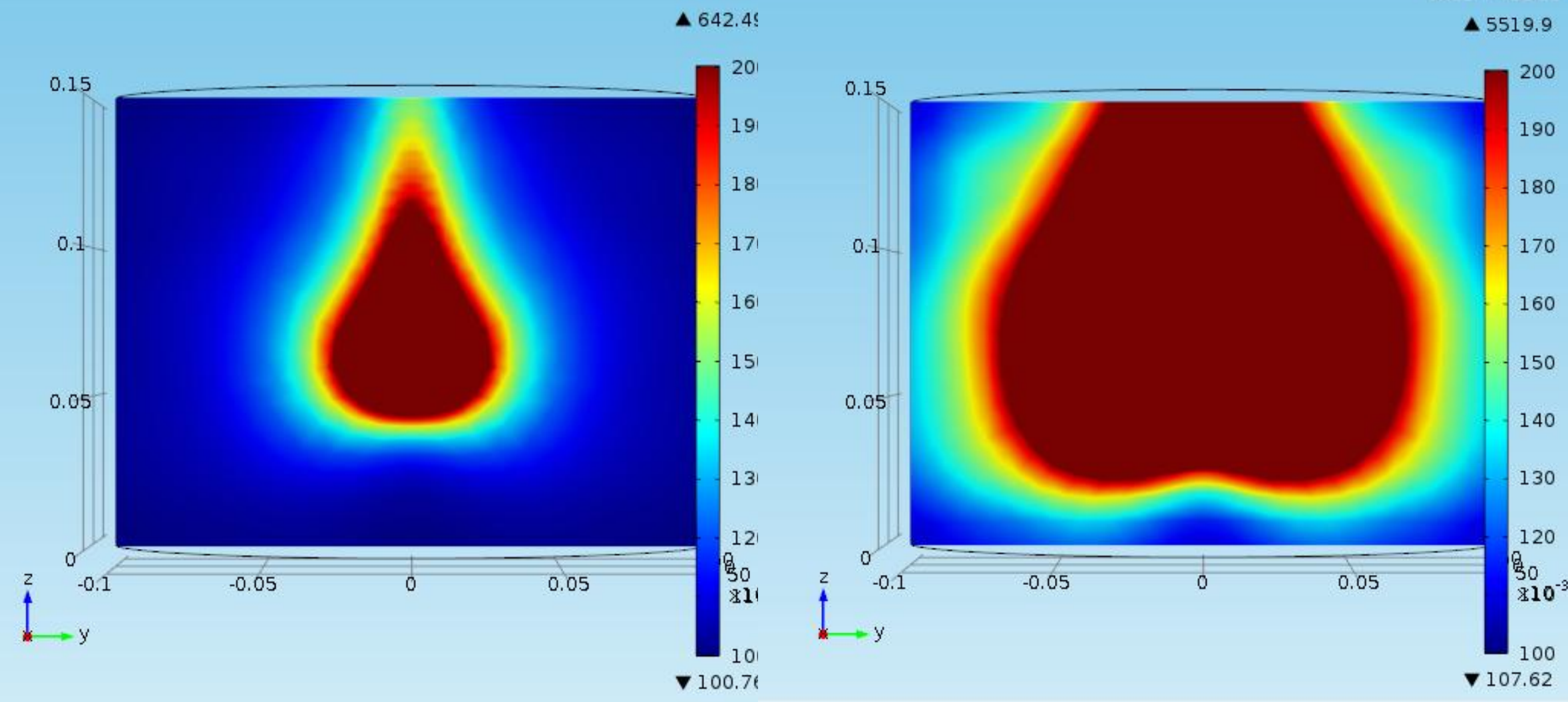




\section{Directional Launcher}

Majority of the power directed in one direction, to the left. Much less power is projected to the $Y$ direction.

To=100K 100 Watt $40 \mathrm{~min}$ IsoTemperature surfaces 100 to $235 \mathrm{~K}$

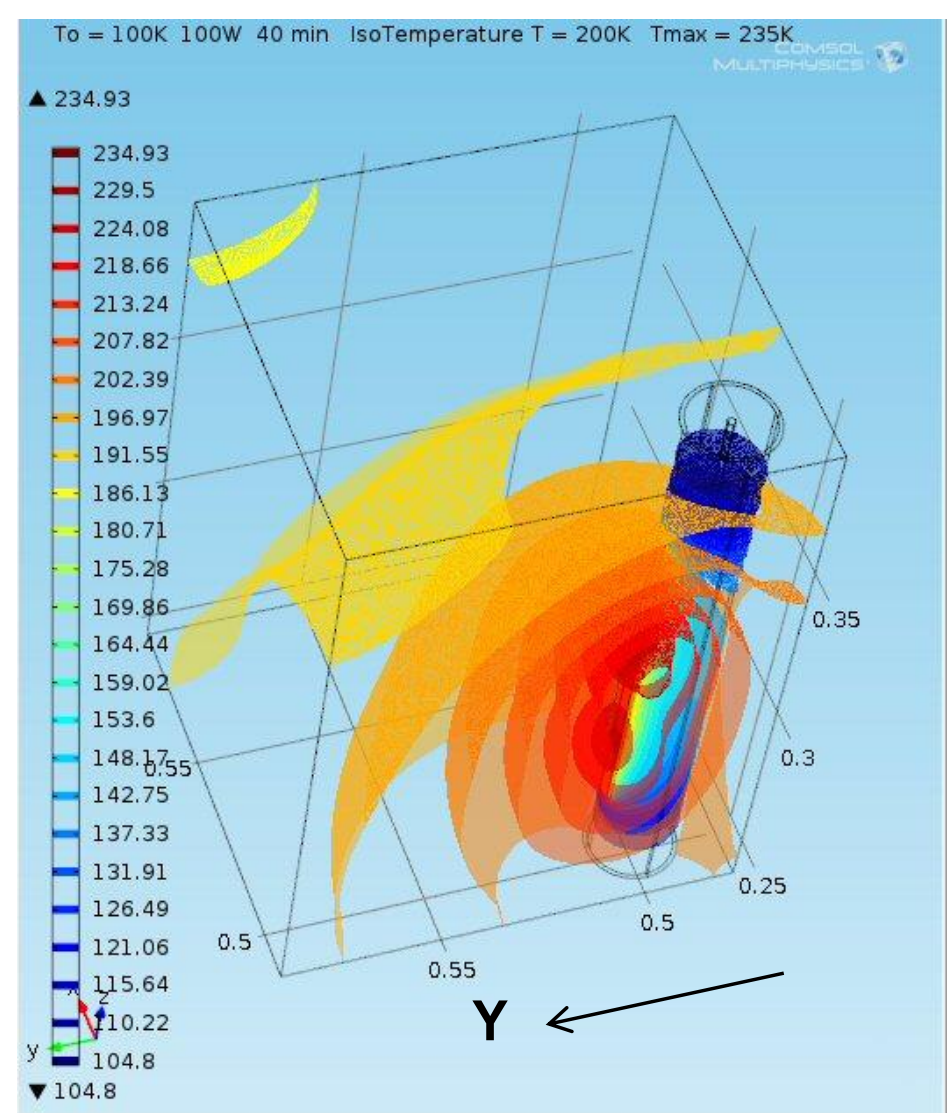

IsoTemperature $=200 \mathrm{~K}$

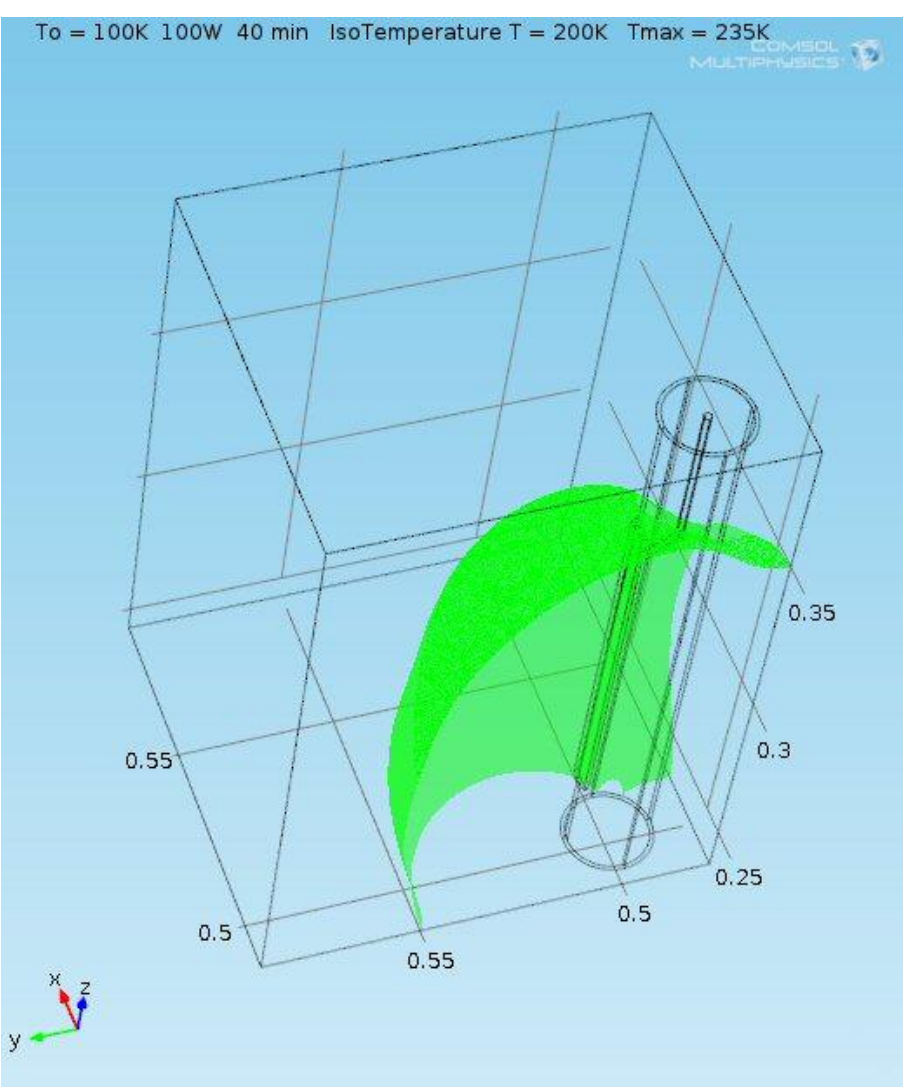




\section{Conclusions}

-COMSOL FEM Multiphysics models have been developed and calculations performed that simulate laboratory experiments. Results are consistent with the observations from the experiments.

- Calculations are consistent with experimental observations, that microwaves $(2.45 \mathrm{GHz})$ will couple with lunar regolith simulants at cryogenic temperatures and heat to temperatures where water ice will sublime at lunar and Martian conditions. This occurs even without the need of "nano-phase metallic iron" in the regolith simulant.

-The range of the dielectric properties of lunar regolith simulants that have been measured have a minor effect on the heating of regolith simulant.

- COMSOL multiphysics is useful for modeling microwave penetration at different microwave frequencies, with different microwave launcher designs and microwave heating. The volume of regolith that is heated sufficiently to completely volatilize the water present in the regolith can be calculated.

- COMSOL will be useful for parametric studies of different scenarios for microwave heating of different planetary surfaces for the design of scientific experiments. It will also permit the design of microwave components and for the determination of hardware requirements for the efficient extraction of water and other volatiles. 


\section{Acknowledgements}

NASA HQ - Support from the Research Opportunities in Space and Earth Sciences (ROSES) - Lunar Advanced Science and Exploration Research (LASER) program.

MSFC Management - Seed Funding

COMSOL - Walter Frei -Technical assistance with the microwave heating models

Contacts:

MSFC Public Affairs - Steve.Roy@msfc.nasa.gov

MSFC Technology Licensing - 256-544-5353

PI - Edwin Ethridge ed.ethridge@nasa.gov 


\section{Backup Slides}

NASA 